\title{
Total Synthesis of ( \pm )-Marsupellins A and B via Acetoxymarsupellone Using an Intramolecular Reductive Cyclization of Epoxycyanohydrin Derivative with $\mathrm{Cp}_{2}$ TiI
}

\author{
Kazuma Matsunaga, Naoki Saito, Hiroshi Kogen, and Kazuhiko Takatori* \\ Graduate School of Pharmaceutical Sciences, Meiji Pharmaceutical University, \\ 2-522-1 Noshio, Kiyose, Tokyo 204-8588, Japan
}

\section{Supporting Information}

Table of Contents

$\begin{array}{lr}\cdot \text { General Information } & \text { S1 } \\ \text { • Experimental Procedures } & \text { S2 } \\ \text { - Comparison of the NMR Spectra of Synthetic and Natural Product } & \text { S16 } \\ \cdot \text { X-ray Crystallographic Analysis } & \mathrm{S} 19 \\ \cdot \text { References } & \mathrm{S} 21 \\ \cdot{ }^{1} \text { H- and }{ }^{13} \text { C-NMR Spectra } & \text { S22 }\end{array}$

\section{General Information}

All reactions were carried out under an inert atmosphere of argon with dry solvents, unless otherwise stated. Reagents of the highest commercial quality were purchased and used without further purification, unless otherwise stated. Anhydrous THF and $\mathrm{CH}_{2} \mathrm{Cl}_{2}$ were purchased from Kanto Chemical Industries Ltd. Other anhydrous solvents and reagents were prepared by standard methods. Reactions were monitored by thin-layer chromatography carried out on Wako silica gel $70 \mathrm{~F}_{254}$ plate and visualized using ultraviolet light and p-anisaldehyde, potassium permanganate, or cerium molybdate stain. ${ }^{1} \mathrm{H}$ - and ${ }^{13} \mathrm{C}-\mathrm{NMR}$ spectra were recorded on a JEOL JNM-ECS400 $\left({ }^{1} \mathrm{H}: 400 \mathrm{MHz} ;{ }^{13} \mathrm{C}: 100\right.$

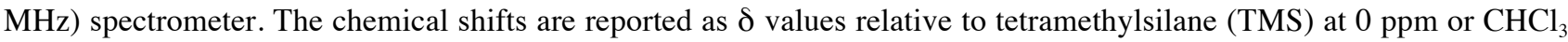
at $7.26 \mathrm{ppm}$ in $\mathrm{CDCl}_{3}$ for ${ }^{1} \mathrm{H}-\mathrm{NMR}$, and relative to $\mathrm{CDCl}_{3}$ at $77.0 \mathrm{ppm}$ for ${ }^{13} \mathrm{C}-\mathrm{NMR}$. In an attribution of proton and carbon by ${ }^{1} \mathrm{H}-\mathrm{NMR}$ and ${ }^{13} \mathrm{C}-\mathrm{NMR}$, the carbon skeleton numbering systems was described according to that for acetoxymarsupellone and 9-acetoxymarsupellol. IR spectra were recorded on a JASCO FT/IR 4100 Series spectrometer. MS spectra were recorded on a JEOL JMS-700 double-focusing spectrometer. Elemental analyses were recorded on a Yanaco CHN CORDER MT-6. Melting points were measured on a Yanaco micro melting point apparatus and are uncorrected. 


\section{Experimental Procedures}

(E)-8-Iodo-5,5-dimethylocta-1,3-diene (10)

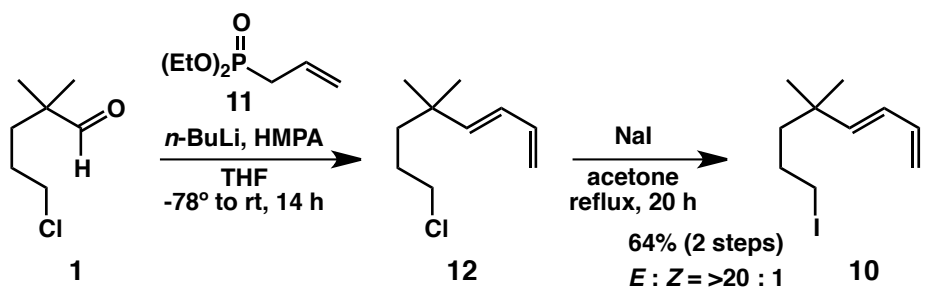

To a solution of $\mathbf{1 1}^{1}$ (17.9. $\left.\mathrm{g}, 101 \mathrm{mmol}\right)$ in THF $(200 \mathrm{~mL})$ was added a solution of $n$-BuLi in hexane $(2.69 \mathrm{M}, 37.4 \mathrm{~mL}$, $101 \mathrm{mmol})$ at $-78{ }^{\circ} \mathrm{C}$ and the mixture was stirred for $15 \mathrm{~min}$. A solution of $\mathbf{1}^{2}(12.5 \mathrm{~g}, 83.8 \mathrm{mmol})$ and $\mathrm{HMPA}(35.1 \mathrm{~mL}$, $202 \mathrm{mmol})$ in THF $(80 \mathrm{~mL})$ was added dropwise and the mixture was stirred for $2 \mathrm{~h}$ at $-78{ }^{\circ} \mathrm{C} .{ }^{3}$ After $14 \mathrm{~h}$ at room temperature, saturated aqueous $\mathrm{NH}_{4} \mathrm{Cl}(100 \mathrm{~mL})$ and water $(100 \mathrm{~mL})$ were added, and the mixture was extracted with $\mathrm{Et}_{2} \mathrm{O}(1 \mathrm{~L})$. The organic layer was washed with aqueous $\mathrm{HCl}(1.0 \mathrm{M}, 50 \mathrm{~mL})$ and brine, dried over $\mathrm{Na}_{2} \mathrm{SO}_{4}$, and concentrated in vacuo.

To the solution of the crude in acetone $(220 \mathrm{~mL})$ was added $\mathrm{NaI}(97.2 \mathrm{~g}, 649 \mathrm{mmol})$ at room temperature. The reaction mixture was warmed up to reflux and stirred for $20 \mathrm{~h}$. After addition of hexane $(40 \mathrm{ml})$, the precipitate was removed by filtration through silica gel and washed (hexane: $\mathrm{Et}_{2} \mathrm{O}=1: 1$ ). The filtrate was concentrated in vacuo. The crude product was purified by column chromatography on silica gel (hexane: $\left.\mathrm{Et}_{2} \mathrm{O}=20: 1\right)$ to give $\mathbf{1 0}$ as a colorless oil $(14.1 \mathrm{~g}, 64 \%$ yield).

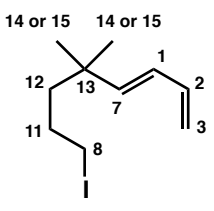

10

${ }^{1} \mathrm{H}-\mathrm{NMR}\left(400 \mathrm{MHz}, \mathrm{CDCl}_{3}\right)$ d: $6.31(1 \mathrm{H}, \mathrm{ddt}, J=16.9,0.7,10.3 \mathrm{~Hz}, \mathrm{C} 2-\mathrm{H}), 5.96(1 \mathrm{H}, \mathrm{dd}, J=15.6,10.3 \mathrm{~Hz}, \mathrm{C} 1-\mathrm{H})$, $5.63(1 \mathrm{H}, \mathrm{dd}, J=15.6,0.5 \mathrm{~Hz}, \mathrm{C} 7-\mathrm{H}), 5.13(1 \mathrm{H}, \mathrm{m}, \mathrm{C} 3-\mathrm{H}), 4.99(1 \mathrm{H}, \mathrm{m}, \mathrm{C} 3-\mathrm{H}), 3.14(2 \mathrm{H}, \mathrm{t}, J=7.0 \mathrm{~Hz}, \mathrm{C} 8-\mathrm{H} \times 2), 1.75$ $(2 \mathrm{H}, \mathrm{m}, \mathrm{C} 11-\mathrm{H} \times 2), 1.39(2 \mathrm{H}, \mathrm{m}, \mathrm{C} 12-\mathrm{H} \times 2), 1.02(6 \mathrm{H}, \mathrm{s}, \mathrm{C} 14 \mathrm{Me}, \mathrm{C} 15 \mathrm{Me})$.

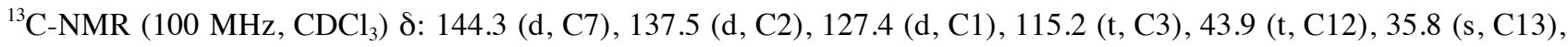
29.1 (t, C11), 27.1 (q, C14, C15), 7.7 (t, C8).

IR (neat): 3033, 2959, 2911, 2867, 2845, 1649, 1470, 1363, 1004, $897 \mathrm{~cm}^{-1}$.

EI-MS m/z (\%): $264\left(\mathrm{M}^{+}, 66\right), 137$ (11), 109 (14), 95 (100), 81 (15), 67 (20), 55 (11), 41 (9).

HR-EI-MS Calcd for $\mathrm{C}_{10} \mathrm{H}_{17} \mathrm{I}: m / z, 264.0375$. Found: 264.0375. 


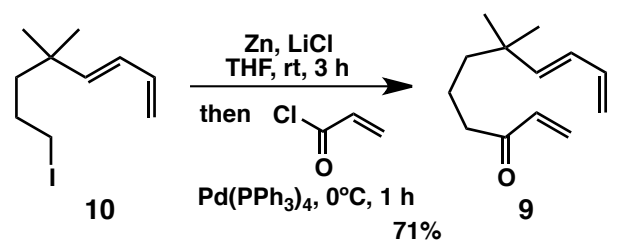

A solution of $\mathbf{1 0}(5.28 \mathrm{~g}, 20.0 \mathrm{mmol})$ in THF (30 mL) was added dropwise to a suspension of $\mathrm{Zn}(2.62 \mathrm{~g}, 40.0 \mathrm{mmol})$ and $\mathrm{LiCl}(848 \mathrm{mg}, 20.0 \mathrm{mmol})$ in THF $(10 \mathrm{~mL})$ at room temperature and the mixture was stirred for $3 \mathrm{~h}$. To a solution of $\mathrm{Pd}\left(\mathrm{PPh}_{3}\right)_{4}(462 \mathrm{~g}, 0.400 \mathrm{mmol})$ in THF $(30 \mathrm{~mL})$ were sequentially added distilled acryloyl chloride $(1.83 \mathrm{~mL}, 2.17 \mathrm{~g}$, $24.0 \mathrm{mmol}$ ) and the above prepared $\mathrm{Zn}$ reagent at $-78^{\circ} \mathrm{C}$. The mixture was stirred for $10 \mathrm{~min}$ at $-78{ }^{\circ} \mathrm{C}$ and for $1 \mathrm{~h}$ at room temperature. Pentane $(200 \mathrm{ml})$ was added to the mixture. The resulting precipitate was removed by filtration through silica gel, washed (pentane: $\mathrm{Et}_{2} \mathrm{O}=1: 1$ ), and the filtrate was concentrated in vacuo. The crude product was purified by column chromatography on silica gel (pentane: $\left.\mathrm{Et}_{2} \mathrm{O}=80: 1-20: 1\right)$ to give 9 as a light yellow oil $(2.74 \mathrm{~g}, 71 \%$ yield).

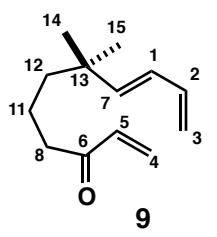

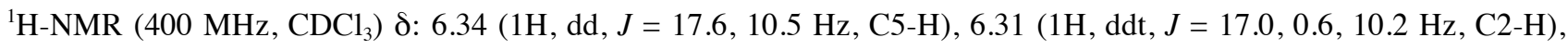
$6.20(1 \mathrm{H}, \mathrm{dd}, J=17.6,10.5 \mathrm{~Hz}, \mathrm{C} 4-\mathrm{H}), 5.97(1 \mathrm{H}, \mathrm{dd}, J=15.6,10.2 \mathrm{~Hz}, \mathrm{C} 1-\mathrm{H}), 5.81(1 \mathrm{H}, \mathrm{dd}, J=10.5,1.2 \mathrm{~Hz}, \mathrm{C} 4-\mathrm{H})$, $5.65(1 \mathrm{H}, \mathrm{dd}, J=15.6,0.6 \mathrm{~Hz}, \mathrm{C} 7-\mathrm{H}), 5.11(1 \mathrm{H}, \mathrm{m}, \mathrm{C} 3-\mathrm{H}), 4.97(1 \mathrm{H}, \mathrm{m}, \mathrm{C} 3-\mathrm{H}), 2.54(2 \mathrm{H}, \mathrm{t}, J=7.3 \mathrm{~Hz}, \mathrm{C} 8-\mathrm{H} \times 2), 1.55$ $(2 \mathrm{H}, \mathrm{m}, \mathrm{C} 11-\mathrm{H} \times 2), 1.30(2 \mathrm{H}, \mathrm{m}, \mathrm{C} 12-\mathrm{H} \times 2), 1.02(6 \mathrm{H}, \mathrm{s}, \mathrm{C} 14 \mathrm{Me}, \mathrm{C} 15 \mathrm{Me})$.

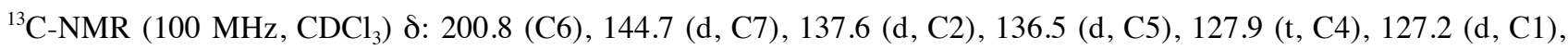
114.9 (t, C3), 42,5 (t, C12), 40.1 (t, C8), 36.0 (s, C13), 27.0 (q, C14, C15), 19.2 (t, C11).

IR (neat): 2959, 2900, 2870, 1700, 1683, 1648, 1615, 1470, 1458, 1401, 1385, 1362, 1200, 1092, 1006, $957,897 \mathrm{~cm}^{-1}$. EI-MS m/z (\%): $192\left(\mathrm{M}^{+}, 21\right), 122$ (39), 107 (81), 95 (100), 79 (39), 67 (26), 55 (44), 41 (15).

HR-EI-MS Calcd for $\mathrm{C}_{13} \mathrm{H}_{20} \mathrm{O}: \mathrm{m} / z$ 192.1514. Found: 192.1516.

$\left(1 S^{*}, 7 R^{*}\right)-6,6-D i m e t h y l b i c y c l o[5.4 .0]$ undec-8-en-2-one (3)

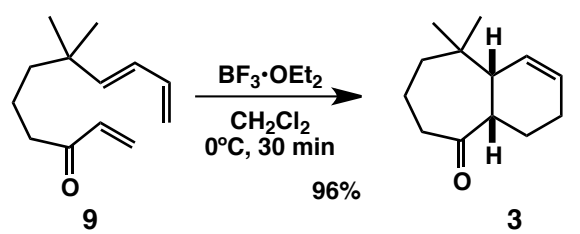

To a solution of $9(154 \mathrm{mg}, 0.800 \mathrm{mmol})$ in $\mathrm{CH}_{2} \mathrm{Cl}_{2}(8 \mathrm{~mL})$ was added distilled $\mathrm{BF}_{3} \cdot \mathrm{OEt}_{2}(0.10 \mathrm{~mL}, 0.11 \mathrm{~g}, 0.80 \mathrm{mmol})$ at $0{ }^{\circ} \mathrm{C}$ and the mixture was stirred for $30 \mathrm{~min}$. The reaction was quenched by addition of saturated aqueous $\mathrm{NaHCO}_{3}(5$ $\mathrm{mL}$ ) and pentane $(50 \mathrm{~mL})$, the organic layer was washed with brine, dried over $\mathrm{Na}_{2} \mathrm{SO}_{4}$, and concentrated in vacuo. The crude product was purified by column chromatography on silica gel (pentane: $\mathrm{Et}_{2} \mathrm{O}=40: 1-20: 1$ ) to give 3 as a colorless oil (148 mg, 96\% yield, dr: >20:1). 


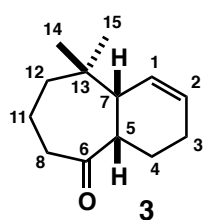

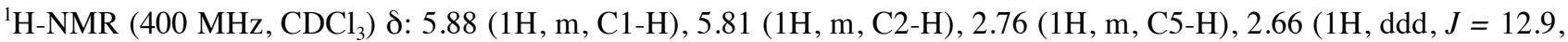
11.2, $2.8 \mathrm{~Hz}, \mathrm{C} 8-\mathrm{H}), 2.39$ (1H, m, C8-H), 2.26 (1H, m, C7-H), 2.07 (1H, m, C4-H), 2.00 (1H, m, C3-H), $1.95-1.80$ (2H, m, C3-H, C4-H), 1.72 (1H, dt, $J=3.7,13.5 \mathrm{~Hz}, \mathrm{C} 12-\mathrm{H}), 1.68(1 \mathrm{H}, \mathrm{m}, \mathrm{C} 11-\mathrm{H}), 1.48(1 \mathrm{H}, \mathrm{m}, \mathrm{C} 11-\mathrm{H}), 1.33(1 \mathrm{H}, \mathrm{m}$, C12-H), 1.07 (3H, s, C15 Me), 0.90 (3H, s, C14 Me).

${ }^{13} \mathrm{C}-\mathrm{NMR}\left(100 \mathrm{MHz}, \mathrm{CDCl}_{3}\right.$ ) $\delta: 217.9$ (s, C6), 128.2 (d, C1), 127.6 (d, C2), 48.4 (d, C5), 44.9 (d, C7), 44.6 (t, C8), 38.2 (t, C12), 36.4 (s, C13), 31.0 (q, C15), 28.4 (t, C4), 25.2 (q, C14), 22.3 (t, C3), 21.7 (t, C11).

IR (neat): 3028, 2957, 2929, 2869, 1692, 1454, 1438, $1133 \mathrm{~cm}^{-1}$.

EI-MS m/z (\%): 192 (M+100), 149 (10), 136 (10), 135 (12), 123 (19), 122 (24), 121 (17), 111 (30), 109 (17), 108 (11), 105 (17), 104 (12), 95 (16), 93 (11), 91 (14), 83 (16), 81 (21), 80 (27), 79 (29), 77 (13), 69 (19), 67 (10), 56 (10), 55 (20), 41 (14). HR-EI-MS Calcd for $\mathrm{C}_{13} \mathrm{H}_{20} \mathrm{O}: \mathrm{m} / z$ 192.1514. Found: 192.1511

\section{$\left(1 S^{*}, 7 R^{*}\right)-6,6-D i m e t h y l b i c y c l o[5.4 .0]$ undec-8-en-2-one (3)}

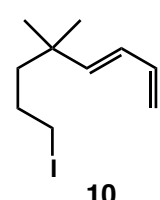

10

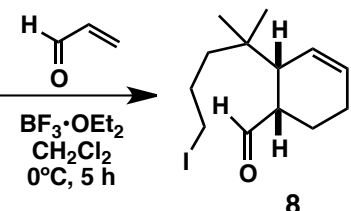

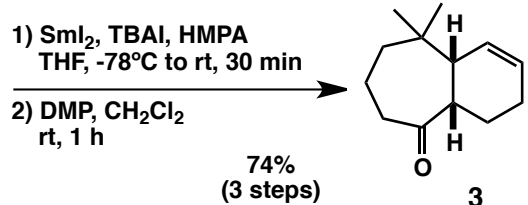

To a solution of $14(2.64 \mathrm{~g}, 10.0 \mathrm{mmol})$ and distilled acrolein $(2.67 \mathrm{~mL}, 2.24 \mathrm{~g}, 40.0 \mathrm{mmol})$ in $\mathrm{CH}_{2} \mathrm{Cl}_{2}(67 \mathrm{~mL})$ was added distilled $\mathrm{BF}_{3} \cdot \mathrm{OEt}_{2}(0.25 \mathrm{~mL}, 0.28 \mathrm{~g}, 2.0 \mathrm{mmol})$ at $0{ }^{\circ} \mathrm{C}$ and the mixture was stirred for $5 \mathrm{~h}$. The reaction was quenched by addition of saturated aqueous $\mathrm{NaHCO}_{3}(50 \mathrm{~mL})$ and hexane: $\mathrm{Et}_{2} \mathrm{O}(=1: 1,500 \mathrm{~mL})$, the organic layer was washed with brine, dried over $\mathrm{Na}_{2} \mathrm{SO}_{4}$, and concentrated in vacuo to give $\mathbf{8}$.

A solution of the crude 8 in THF $(100 \mathrm{~mL})$ was added dropwise to a stirred solution of $\mathrm{SmI}_{2}$ in THF $(0.1 \mathrm{M}, 300 \mathrm{~mL}$, $30.0 \mathrm{mmol})$, TBAI $(22.2 \mathrm{~g}, 60.0 \mathrm{mmol})$ and HMPA $(20.9 \mathrm{~mL}, 21.5 \mathrm{~g}, 120 \mathrm{mmol})$ at $-78{ }^{\circ} \mathrm{C} .{ }^{4 \mathrm{a}}$ The mixture was stirred for $10 \mathrm{~min}$ at $-78{ }^{\circ} \mathrm{C}$ and for $30 \mathrm{~min}$ at room temperature. The reaction was quenched by blowing air into the flask while stirring the mixture. The mixture was diluted with excess $\mathrm{Et}_{2} \mathrm{O}$. While the mixture was vigorously stirred, saturated aqueous $\mathrm{NaHCO}_{3}$ and $20 \%$ aqueous $\mathrm{Na}_{2} \mathrm{~S}_{2} \mathrm{O}_{3}$ solution were sequentially added dropwise at a volume ratio of $2: 1{ }^{4}$ The resulting precipitate was separated by filtration through silica gel, and the filtrate was concentrated in vacuo.

To the solution of the crude alcohols in $\mathrm{CH}_{2} \mathrm{Cl}_{2}(100 \mathrm{~mL})$ was added DMP $(6.36 \mathrm{~g}, 15.0 \mathrm{mmol})$ at room temperature and the mixture was stirred for $1 \mathrm{~h}$. The reaction was quenched by addition of a $20 \%$ aqueous $\mathrm{Na}_{2} \mathrm{~S}_{2} \mathrm{O}_{3}(20 \mathrm{~mL})$, saturated aqueous $\mathrm{NaHCO}_{3}(20 \mathrm{~mL})$ and pentane $(500 \mathrm{~mL})$. After stirring for $30 \mathrm{~min}$, the organic layer was washed with saturated aqueous $\mathrm{NaHCO}_{3}$ and brine, dried over $\mathrm{Na}_{2} \mathrm{SO}_{4}$, and concentrated in vacuo. The crude product was purified by column chromatography on silica gel (pentane: $\mathrm{Et}_{2} \mathrm{O}=40: 1-20: 1$ ) to give 3 as a colorless oil (1.42 g, 74\% yield). 


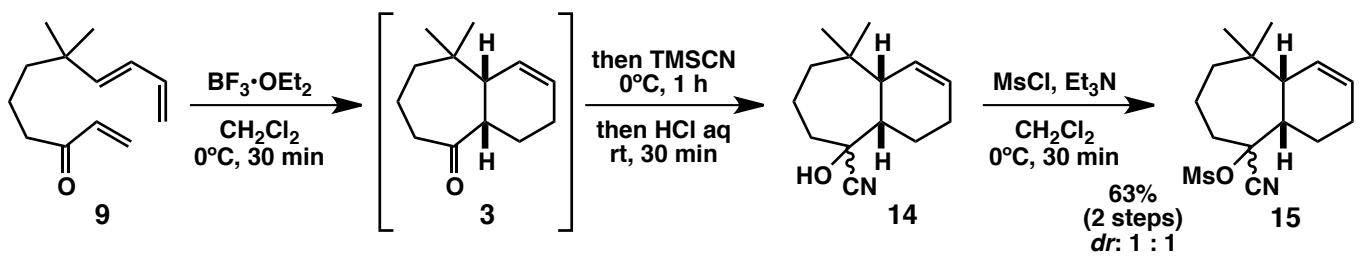

To a solution of $9(1.50 \mathrm{~g}, 7.80 \mathrm{mmol})$ in $\mathrm{CH}_{2} \mathrm{Cl}_{2}(78 \mathrm{~mL})$ was added distilled $\mathrm{BF}_{3} \cdot \mathrm{OEt}_{2}(0.98 \mathrm{~mL}, 1,1 \mathrm{~g}, 7.80 \mathrm{mmol})$ at $0{ }^{\circ} \mathrm{C}$ and the mixture was stirred for $30 \mathrm{~min}$. TMSCN $(0.98 \mathrm{~mL}, 0.77 \mathrm{~g}, 7.8 \mathrm{mmol})$ was added at $0{ }^{\circ} \mathrm{C}$ and the mixture was stirred for $1 \mathrm{~h}$. Aqueous $\mathrm{HCl}(1.0 \mathrm{M}, 50 \mathrm{~mL})$ was added at $0{ }^{\circ} \mathrm{C}$ and the mixture was stirred for $1 \mathrm{~h}$ at room temperature. The reaction mixture was diluted with $\mathrm{Et}_{2} \mathrm{O}(400 \mathrm{~mL})$. The organic layer was separated, washed with brine, dried over $\mathrm{Na}_{2} \mathrm{SO}_{4}$, and concentrated in vacuo to give 14 .

To the solution of the crude 14 were added $\mathrm{MsCl}(0.91 \mathrm{~mL}, 1.3 \mathrm{~g}, 0.11 \mathrm{mmol})$ and $\mathrm{Et}_{3} \mathrm{~N}(1.30 \mathrm{~mL}, 947 \mathrm{mg}, 9.36 \mathrm{mmol})$ at $-78^{\circ} \mathrm{C}$. After stirred for $30 \mathrm{~min}$ at $0^{\circ} \mathrm{C}$, the reaction was quenched by addition of water $(30 \mathrm{~mL})$ and $\mathrm{Et}_{2} \mathrm{O}(400 \mathrm{~mL})$. The organic layer was washed aqueous $\mathrm{HCl}(1.0 \mathrm{M})$ and brine, dried over $\mathrm{Na}_{2} \mathrm{SO}_{4}$, and concentrated in vacuo. The crude product was purified by column chromatography on silica gel (hexane:AcOEt $=8: 1-4: 1$ ) to give $\mathbf{1 5}$ as a colorless oil (1.46 g, 63\% yield).

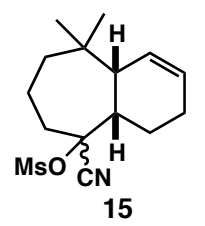

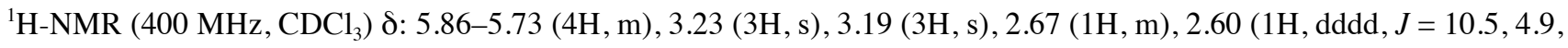
3.2, $0.7 \mathrm{~Hz}), 2.52(1 \mathrm{H}, \mathrm{dddd}, J=14.9,6.3,5.4,0.9 \mathrm{~Hz}), 2.46-2.12(9 \mathrm{H}, \mathrm{m}), 2.02-1.64(10 \mathrm{H}, \mathrm{m}), 1.59-1.50(2 \mathrm{H}, \mathrm{m})$, $1.04(3 \mathrm{H}, \mathrm{s}), 1.04(3 \mathrm{H}, \mathrm{s}), 0.96(3 \mathrm{H}, \mathrm{s}), 0.94(3 \mathrm{H}, \mathrm{s})$.

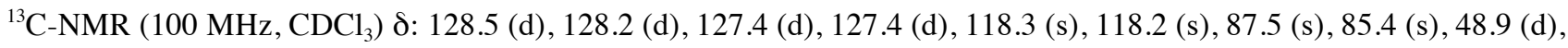
$47.5(\mathrm{~d}), 44.9$ (d), 44.8 (d), $40.3(\mathrm{~d}), 40.2(\mathrm{~d}), 40.1(\mathrm{t}), 39.7$ (t), $37.9(\mathrm{~s}), 37.9(\mathrm{~s}), 33.6(\mathrm{t}), 33.2(\mathrm{q}), 33.0(\mathrm{t}), 31.8(\mathrm{q}), 25.7$ $(\mathrm{t}), 25.3(\mathrm{q}), 25.2(\mathrm{t}), 25.0(\mathrm{q}), 23.3(\mathrm{t}), 19.9(\mathrm{t}), 19.2(\mathrm{t}), 18.3(\mathrm{t})$.

IR (neat): $3031,2955,2871,2205,1463,1364,1180,1092,912,876 \mathrm{~cm}^{-1}$.

EI-MS m/z (\%): 297 (M+, 0.3), 218 (69), 201 (100), 186 (54), 173 (12), 158 (28), 145 (16), 131 (66), 121 (14), 117 (25), 105 (18), 95 (12), 91 (18), 79 (36), 69 (81), 55 (23), 41 (25).

HR-EI-MS Calcd for $\mathrm{C}_{15} \mathrm{H}_{23} \mathrm{NO}_{3} \mathrm{~S}: \mathrm{m} / z$ 297.1399. Found: 297.1398.

$\left(1 S^{*}, 2 S^{*}, 7 R^{*}, 8 R^{*}, 9 S^{*}\right)-2-C y a n o-8,9-e p o x y-6,6-d i m e t h y l b i c y c l o[5.4 .0]$ undec-2-yl methanesulfonate $(7 \alpha)$ $\left(1 S^{*}, 2 R^{*}, 7 R^{*}, 8 R^{*}, 9 S^{*}\right)-2-C y a n o-8,9-e p o x y-6,6-d i m e t h y l b i c y c l o[5.4 .0]$ undec-2-yl methanesulfonate (7ß)
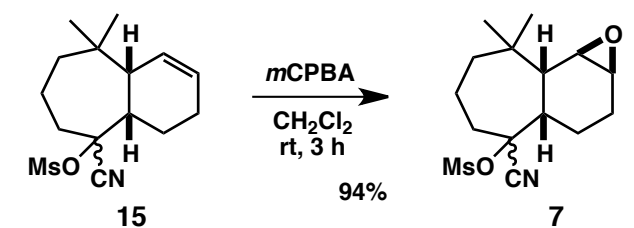

To a solution of $3(941 \mathrm{mg}, 3.16 \mathrm{mmol})$ in $\mathrm{CH}_{2} \mathrm{Cl}_{2}(16 \mathrm{~mL})$ was added $m \mathrm{CPBA}(77 \%, 1.06 \mathrm{~g}, 4.75 \mathrm{mmol})$ at $0{ }^{\circ} \mathrm{C}$. The reaction mixture was warmed up to room temperature and stirred for $3 \mathrm{~h}$. The reaction was quenched by addition of saturated aqueous $\mathrm{NaHCO}_{3}(20 \mathrm{~mL})$, a $20 \%$ aqueous $\mathrm{Na}_{2} \mathrm{~S}_{2} \mathrm{O}_{3}(10 \mathrm{~mL})$ and $\mathrm{Et}_{2} \mathrm{O}(400 \mathrm{~mL})$. After stirring for $30 \mathrm{~min}$, the 
organic layer was washed with saturated aqueous $\mathrm{NaHCO}_{3}$ and brine, dried over $\mathrm{Na}_{2} \mathrm{SO}_{4}$, and concentrated in vacuo. The crude product was purified by column chromatography on silica gel (hexane:AcOEt $=4: 1-1: 1)$ to give $7(\mathbf{7} \alpha: 7 \beta=1: 1)$ as a colorless oil (934 $\mathrm{mg}, 94 \%$ yield).

Part of 7 was purified by column chromatography on silica gel (hexane:AcOEt $=4: 1-1: 1$ ) and divided $7 \boldsymbol{\alpha}$ and $\mathbf{7} \beta$.

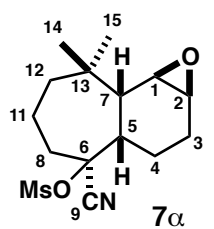

Low polar compound $\mathbf{7} \boldsymbol{\alpha}$.

${ }^{1} \mathrm{H}-\mathrm{NMR}\left(400 \mathrm{MHz}, \mathrm{CDCl}_{3}\right)$ d: 3.30 (1H, m, C1-H), $3.24(1 \mathrm{H}, \mathrm{m}, \mathrm{C} 2-\mathrm{H}), 3.21(3 \mathrm{H}, \mathrm{s}, \mathrm{Ms}), 2.74(1 \mathrm{H}, \mathrm{m}, \mathrm{C}-\mathrm{H}), 2.68$ $(1 \mathrm{H}, \mathrm{m}, \mathrm{C} 8-\mathrm{H}), 2.25(1 \mathrm{H}, \mathrm{t}, J=2.7 \mathrm{~Hz}, \mathrm{C} 7-\mathrm{H}), 2.19(1 \mathrm{H}, \mathrm{dt}, J=16.6,8.4 \mathrm{~Hz}, \mathrm{C} 3-\mathrm{H}), 2.16-2.04(2 \mathrm{H}, \mathrm{m}, \mathrm{C} 3-\mathrm{H}, \mathrm{C} 8-\mathrm{H})$, 1.91-1.58 (6H, m, C4-H ×2, C12-H ×2, C11-H ×2), $1.14(3 \mathrm{H}, \mathrm{s}, \mathrm{C} 14 \mathrm{Me}$ or $\mathrm{C} 15 \mathrm{Me}), 1.12(3 \mathrm{H}, \mathrm{s}, \mathrm{C} 14 \mathrm{Me}$ or C15 Me). ${ }^{13} \mathrm{C}-\mathrm{NMR} \quad\left(100 \mathrm{MHz}, \mathrm{CDCl}_{3}\right)$ 8: 117.9 (s, C9), 85.2 (s, C6), 55.1 (d, C1), 52.1 (d, C2), 42.1 (d, C5), 41.5 (d, C7), 40.8 (t, C11), 40.3 (q, Ms), 36.8 (t, C8), 36.4 (s, C13), 30.6 (q, C14 or C15), 28.3 (q, C14 or C15), 21.5 (t, C3), 20.3 (t, C4), $18.1(\mathrm{t}, \mathrm{C} 12)$.

IR (KBr): 2960, 2905, 1468, 1362, 1177, 923, 882, 822, 801, $780 \mathrm{~cm}^{-1}$.

HR-FAB-MS ( $m$-nitrobenzyl alcohol) Calcd for $\mathrm{C}_{15} \mathrm{H}_{24} \mathrm{NO}_{4} \mathrm{~S}: m / z$ 314.1426. Found: $314.1427\left(\mathrm{MH}^{+}\right)$.

Anal. Calcd for $\mathrm{C}_{15} \mathrm{H}_{23} \mathrm{NO}_{4} \mathrm{~S}$ : C, 57.48; H, 7.40; N, 4.47. Found: C, 57.45; H, 7.28; N, 4.45.

mp. $123-125^{\circ} \mathrm{C}$ (crystallized from hexane and AcOEt).

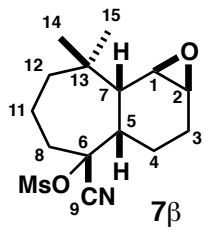

High polar compound $7 \beta$.

${ }^{1} \mathrm{H}-\mathrm{NMR}\left(400 \mathrm{MHz}, \mathrm{CDCl}_{3}\right)$ d: $3.35(1 \mathrm{H}, \mathrm{ddd}, J=3.7,2.9,1.0 \mathrm{~Hz}, \mathrm{C} 1-\mathrm{H}), 3.21(1 \mathrm{H}, \mathrm{t}, J=3.7 \mathrm{~Hz}, \mathrm{C} 2-\mathrm{H}), 3.18(3 \mathrm{H}, \mathrm{s}$, Ms), $2.70(1 \mathrm{H}, \mathrm{m}, \mathrm{C} 5-\mathrm{H}), 2.61(1 \mathrm{H}, \mathrm{m}, \mathrm{C} 8-\mathrm{H}), 2.34(1 \mathrm{H}, \mathrm{t}, J=2.9 \mathrm{~Hz}, \mathrm{C} 7-\mathrm{H}), 2.16(1 \mathrm{H}, \mathrm{ddd}, J=14.0,8.1,4.8 \mathrm{~Hz}$, C8-H), $2.12(1 \mathrm{H}, \mathrm{dt}, J=15.8,8.1 \mathrm{~Hz}, \mathrm{C} 3-\mathrm{H}), 2.02(1 \mathrm{H}, \mathrm{ddt}, J=15.8,8.1,3.7 \mathrm{~Hz}, \mathrm{C} 3-\mathrm{H}), 1.89-1.59(6 \mathrm{H}, \mathrm{m}, \mathrm{C} 12-\mathrm{H} \times 2$, $\mathrm{C} 4-\mathrm{H} \times 2, \mathrm{C} 11-\mathrm{H} \times 2), 1.16$ (3H, s, C14 Me or C15 Me), 1.12 (3H, s, C14 Me or C15 Me).

${ }^{13} \mathrm{C}-\mathrm{NMR}\left(100 \mathrm{MHz}, \mathrm{CDCl}_{3}\right.$ ) ): 117.4 (s, C9), 86.4 (s, C6), 55.5 (d, C1), 52.0 (d, C2), 41.9 (d, C5), 41.8 (d, C7), 40.9 (t, C11), 40.3 (q, Ms), 36.3 (s, C13), 36.1 (t, C8), 31.6 (q, C14 or C15), 27.9 (q, C14 or C15), 21.9 (t, C3), 18.3 (t, C12), $17.9(\mathrm{t}, \mathrm{C} 4)$.

IR (KBr): 2968, 2939, 2918, 2872, 1473, 1358, 1173, 962, 931, 914, 893, 869, 812, 803, $775 \mathrm{~cm}^{-1}$.

HR-FAB-MS ( $m$-nitrobenzyl alcohol) Calcd for $\mathrm{C}_{15} \mathrm{H}_{24} \mathrm{NO}_{4} \mathrm{~S}: m / z 314.1426$. Found: $314.1420\left(\mathrm{MH}^{+}\right)$. 


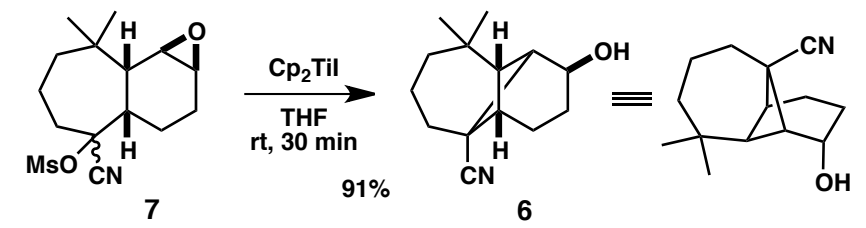

A solution of 7 (934 mg, $2.98 \mathrm{mmol})$ in THF (30 mL) was added dropwise to a stirred solution of Cp $\mathrm{p}_{2} \mathrm{TiI}(0.2 \mathrm{M}, 44.7$ $\mathrm{mL}, 8.94 \mathrm{mmol}$ ) that was prepared in advance by collecting the supernatant of a mixture of $\mathrm{Cp}_{2} \mathrm{TiI}_{2}{ }^{5}$ and $\mathrm{Mn}$ in $\mathrm{THF}^{4 a}$ After stirred for $30 \mathrm{~min}$ at room temperature, the reaction was quenched by blowing air into the flask while stirring the mixture. The mixture was diluted with excess $\mathrm{Et}_{2} \mathrm{O}$. While the mixture was vigorously stirred, saturated aqueous $\mathrm{NaHCO}_{3}$ solution was sequentially added dropwise. The resulting precipitate was separated by filtration through silica gel, and the filtrate was concentrated in vacuo. The crude product was purified by column chromatography on silica gel (hexane:AcOEt $=4: 1)$ to give 6 as a colorless oil $(592 \mathrm{mg}, 91 \%$ yield).

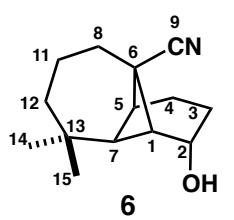

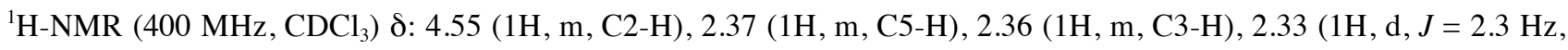
C1-H), 2.17-1.99 (2H, m, C4-H ×2), $2.03(2 \mathrm{H}, \mathrm{t}, J=6.1 \mathrm{~Hz}, \mathrm{C} 8-\mathrm{H} \times 2), 1.75(1 \mathrm{H}, \mathrm{s}, \mathrm{C} 7-\mathrm{H}), 1.72-1.59(3 \mathrm{H}, \mathrm{m}, \mathrm{C} 11-\mathrm{H} \times 2$, C3-H), 1.46-1.40 (2H, m, C12-H ×2), 0.93 (3H, s, C14 Me or C15 Me), 0.91 (3H, s, C14 Me or C15 Me).

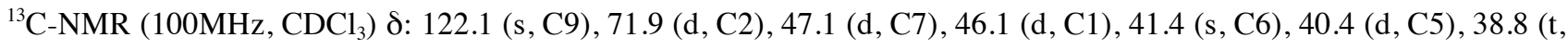
C12), 35.5 (t, C8), 32.0 (s, C13), 27.7 (q, C14 or C15), 27.2 (q, C14 or C15), 27.1 (t, C4), 25.6 (t, C3), 21.0 (t, C11).

IR (neat): 3433, 2951, 2869, 2222, 1466, 1456, 1364, 1273, 1074, 1062, 1012, $956 \mathrm{~cm}^{-1}$.

EI-MS m/z (\%): 219 (M+, 19), 204 (16), 201 (18), 190 (12), 186 (32), 176 (40), 172 (18), 162 (24), 158 (34), 148 (100), 145 (31), 133 (39), 120 (27), 108 (27), 97 (32), 91 (20), 81 (22), 69 (22), 55 (24), 41 (30).

HR-EI-MS Calcd for $\mathrm{C}_{14} \mathrm{H}_{21} \mathrm{NO}: \mathrm{m} / z$ 219.1623. Found: 219.1621.

$\left(1 S^{*}, 2 S^{*}, 7 S^{*}, 8 R^{*}\right)-6,6-D i m e t h y l-9-0 x o t r i c y c l o\left[5.4 .0 .0^{2,8}\right]$ undecane-2-carbonitrile (16)

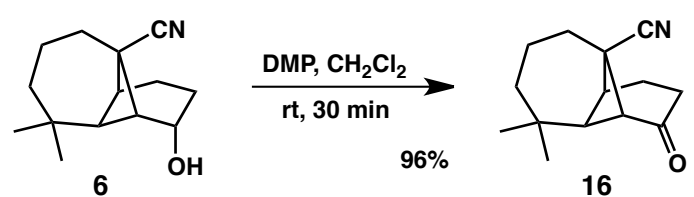

To the solution of $6(515 \mathrm{mg}, 2.35 \mathrm{mmol})$ in $\mathrm{CH}_{2} \mathrm{Cl}_{2}(12 \mathrm{~mL})$ was added DMP $(1.49 \mathrm{~g}, 3.52 \mathrm{mmol})$ at room temperature and the mixture was stirred for $30 \mathrm{~min}$. The reaction was quenched by addition of saturated aqueous $\mathrm{NaHCO}_{3}(20 \mathrm{~mL}), 20 \%$ aqueous $\mathrm{Na}_{2} \mathrm{~S}_{2} \mathrm{O}_{3}(10 \mathrm{~mL})$, and $\mathrm{Et}_{2} \mathrm{O}(100 \mathrm{~mL})$. After stirring for $30 \mathrm{~min}$, the organic layer was washed with saturated aqueous $\mathrm{NaHCO}_{3}$ and brine, dried over $\mathrm{Na}_{2} \mathrm{SO}_{4}$, and concentrated in vacuo. The crude product was purified by column chromatography on silica gel (hexane:AcOEt $=4: 1)$ to give 16 as a white solid $(489 \mathrm{mg}, 96 \%$ yield). 


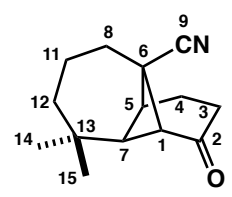

16

${ }^{1} \mathrm{H}-\mathrm{NMR}\left(400 \mathrm{MHz}, \mathrm{CDCl}_{3}\right) \delta: 3.01(1 \mathrm{H}, \mathrm{d}, J=5.3 \mathrm{~Hz}, \mathrm{C} 1-\mathrm{H}), 2.82(1 \mathrm{H}, \mathrm{ddd}, J=20.0,10.2,3.8 \mathrm{~Hz}, \mathrm{C} 3-\mathrm{H}), 2.72(1 \mathrm{H}$, m, C5-H), 2.69 (1H, ddd, $J=20.0,10.8,4.8 \mathrm{~Hz}, \mathrm{C} 3-\mathrm{H}), 2.35$ (1H, dddd, $J=13.5,10.2,4.8,2.9 \mathrm{~Hz}, \mathrm{C} 4-\mathrm{H}), 2.18-2.06$ $(3 \mathrm{H}, \mathrm{m}, \mathrm{C} 8-\mathrm{H} \times 2, \mathrm{C} 4-\mathrm{H}), 1.83-1.69(2 \mathrm{H}, \mathrm{m}, \mathrm{C} 11-\mathrm{H} \times 2), 1.67(1 \mathrm{H}, \mathrm{s}, \mathrm{C} 7-\mathrm{H}), 1.57-1.42(2 \mathrm{H}, \mathrm{m}, \mathrm{C} 12-\mathrm{H} \times 2), 0.96(3 \mathrm{H}, \mathrm{s}$, $\mathrm{C} 14 \mathrm{Me}$ or $\mathrm{C} 15 \mathrm{Me}), 0.91$ (3H, s, C14 Me or C15 Me).

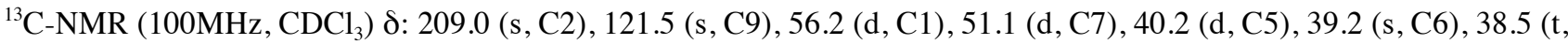
C12), 35.3 (t, C8), 33.0 (s, C13), 32.0 (t, C3), 27.3 (q, C14 or C15), 27.0 (q, C14 or C15), 24.1 (t, C4), 20.8 (t, C11). IR (KBr): 2950, 2868, 2227, 1719, 1459, 1408, 1363, 1302, 1265, 1219, 1159, 1115, $1046 \mathrm{~cm}^{-1}$.

EI-MS m/z (\%): 217 (M+ 90), 202 (30), 199 (10), 190 (55), 176 (63), 161 (50), 149 (66), 146 (56), 133 (77), 119 (41), 111 (100), 106 (132), 95 (39), 91 (39), 83 (76), 77 (33), 69 (51), 55 (52), 41 (55).

HR-EI-MS Calcd for $\mathrm{C}_{14} \mathrm{H}_{19} \mathrm{NO}: m / z$ 217.1467. Found: 217.1465.

\section{$\left(1 S^{*}, 2 S^{*}, 7 S^{*}, 8 S^{*}\right)$-6,6-Dimethyl-9-methylenetricyclo[5.4.0.0 $\left.{ }^{2,8}\right]$ undecane-2-carbonitrile (17)}

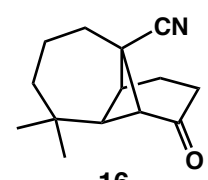

16

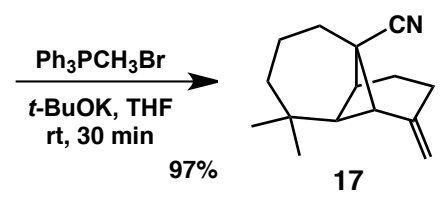

17

A solution of $16(179 \mathrm{mg}, 0.824 \mathrm{mmol})$ in THF $(4.2 \mathrm{~mL})$ was added dropwise to a stirred suspension of Wittig reagent prepared by methyltriphenylphosphonium bromide $(2.94 \mathrm{~g}, 0.824 \mathrm{mmol})$ and $t$-BuOK (462 mg, $4.12 \mathrm{mmol})$ in THF (4.2 $\mathrm{mL}$ ) at room temperature. After stirred for $30 \mathrm{~min}$, the reaction was quenched by addition of saturated aqueous $\mathrm{NH}_{4} \mathrm{Cl}$ $(10 \mathrm{~mL})$ and hexane $(100 \mathrm{~mL})$. The organic layer was washed with brine, dried over $\mathrm{Na}_{2} \mathrm{SO}_{4}$, and concentrated in vacuo. The crude product was purified by column chromatography on silica gel (hexane:Et ${ }_{2} \mathrm{O}=5: 1$ ) to give 17 as a white solid (171 mg, 97\% yield).

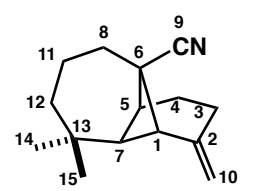

17

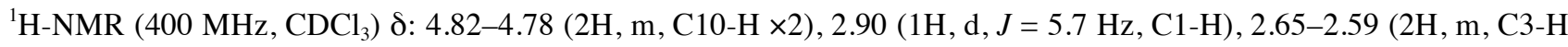
×2), $2.46(1 \mathrm{H}, \mathrm{m}, \mathrm{C} 5-\mathrm{H}), 2.30-2.20(1 \mathrm{H}, \mathrm{m}, \mathrm{C} 4-\mathrm{H}), 2.09-1.99(3 \mathrm{H}, \mathrm{m}, \mathrm{C} 4-\mathrm{H}, \mathrm{C} 8-\mathrm{H} \times 2), 1.73-1.65(2 \mathrm{H}, \mathrm{m}, \mathrm{C} 11-\mathrm{H} \times 2)$, $1.61(1 \mathrm{H}, \mathrm{s}, \mathrm{C} 7-\mathrm{H}), 1.47-1.42(2 \mathrm{H}, \mathrm{m}, \mathrm{C} 12-\mathrm{H} \times 2), 0.91$ (3H, s, C14 Me or C15 Me), 0.90 (3H, s, C14 Me or C15 Me).

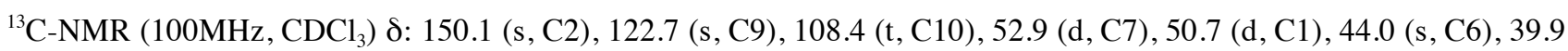
(d, C5), 38.8 (t, C12), 35.2 (t, C8), 32.6 (s, C13), 27.5 (q, C14 or C15), 27.5 (q, C14 or C15), 27.5 (t, C4), 22.9 (t, C3), $21.0(\mathrm{t}, \mathrm{C} 11)$.

IR (KBr): 3080, 2949, 2932, 2868, 2226, 1640, 1457, 1363, 1274, 1008, 890, $\mathrm{cm}^{-1}$.

EI-MS m/z (\%): 215 (M+ $\left.\mathrm{M}^{+}, 82\right), 200$ (100), 186 (28), 172 (55), 159 (34), 145 (34), 131 (45), 121 (25), 117 (20), 108 (74), 96 (22), 93 (55), 80 (31), 77 (26), 69 (16), 55 (19), 41 (27).

HR-EI-MS Calcd for $\mathrm{C}_{15} \mathrm{H}_{21} \mathrm{~N}: m / z$ 215.1674. Found: 215.1677. 
Anal. Calcd for $\mathrm{C}_{15} \mathrm{H}_{21} \mathrm{~N}$ : C, 83.67; H, 9.83; N, 6.50. Found: C, 83.76; H, 9.77; N, 6.55.

mp. $81-83^{\circ} \mathrm{C}$ (crystallized from hexane).

$\left[\left(1 S^{*}, 2 S^{*}, 7 S^{*}, 8 S^{*}\right)-6,6-D i m e t h y l-9-m e t h y l e n e t r i c y c l o\left[5.4 .0 .0^{2,8}\right]\right.$ undecan-2-yl]methanol (18)

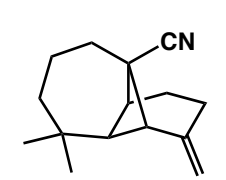

17

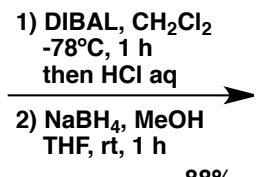

(2 steps)

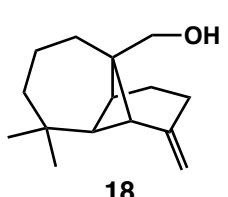

18

To a solution of $17(110 \mathrm{mg}, 0.511 \mathrm{mmol})$ in $\mathrm{CH}_{2} \mathrm{Cl}_{2}(5.1 \mathrm{~mL})$ was added a solution of DIBAL in toluene $(1.01 \mathrm{M}, 0.61$ $\mathrm{mL}, 0.613 \mathrm{mmol})$ at $-78{ }^{\circ} \mathrm{C}$, and the mixture was stirred for $1 \mathrm{~h}$. Excess $\mathrm{Et}_{2} \mathrm{O}$ was added to dilute and brine was added. The mixture was vigorously stirred until the supernatant was clear. The solution was washed with $1 \mathrm{M} \mathrm{HCl}$ aq. and brine, dried over $\mathrm{Na}_{2} \mathrm{SO}_{4}$, and concentrated in vacuo.

The resulting residue was disolved in THF $(2.4 \mathrm{~mL})$. $\mathrm{MeOH}(0.24 \mathrm{~mL})$ and $\mathrm{NaBH}_{4}(19.3 \mathrm{mg}, 0.511$ mmol) were added. The mixture was stirred for $1 \mathrm{~h}$ at room temperature, diluted with $\mathrm{Et}_{2} \mathrm{O}$, washed with $1 \mathrm{M} \mathrm{HCl}$ aq. and brine, dried over $\mathrm{Na}_{2} \mathrm{SO}_{4}$, and concentrated in vacuo. The crude product was purified by column chromatography on silica gel (hexane: $\left.\mathrm{Et}_{2} \mathrm{O}=10: 1-4: 1\right)$ to give $\mathbf{1 8}$ as a white solid (99.1 $\mathrm{mg}, 88 \%$ yield).

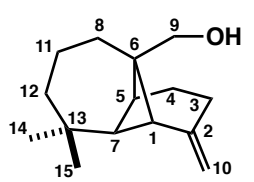

18

${ }^{1} \mathrm{H}-\mathrm{NMR}\left(400 \mathrm{MHz}, \mathrm{CDCl}_{3}\right)$ ঠ: 4.66-4.62 (2H, m, C10-H ×2), $3.46(1 \mathrm{H}, \mathrm{d}, J=11.3 \mathrm{~Hz}, \mathrm{C} 9-\mathrm{H}), 3.25(1 \mathrm{H}, \mathrm{d}, J=11.3 \mathrm{~Hz}$, C9-H), 2.69 (1H, d, J = 5.5 Hz, C1-H), 2.65 (1H, m, C3-H), 2.38 (1H, m, C3-H), 2.18 (1H, m, C5-H), 1.94 (1H, dddd, J $=13.3,9.1,6.8,2.2 \mathrm{~Hz}, \mathrm{C} 4-\mathrm{H}), 1.90-1.80(2 \mathrm{H}, \mathrm{m}, \mathrm{C} 4-\mathrm{H}, \mathrm{C} 8-\mathrm{H}), 1.68-1.59(3 \mathrm{H}, \mathrm{m}, \mathrm{C} 8-\mathrm{H}, \mathrm{C} 11-\mathrm{H} \times 2), 1.53(1 \mathrm{H}, \mathrm{s}$, C7-H), 1.45-1.39 (2H, m, C12-H ×2), 0.91 (3H, s, C14 Me or C15 Me), 0.90 (3H, s, C14 Me or C15 Me).

${ }^{13} \mathrm{C}-\mathrm{NMR}\left(100 \mathrm{MHz}, \mathrm{CDCl}_{3}\right.$ ) $\delta: 153.6$ (s, C2), 105.5 (t, C10), 68.2 (t, C9), 51.6 (d, C7), 48.3 (d, C1), 45.8 (s, C6), 39.6 (t, C12), 38.0 (d, C5), 35.5 (t, C8), 32.6 (s, C13), 28.2 (q, C14 or C15), 27.7 (q, C14 or C15), 25.2 (t, C4), 24.1 (t, C3), 21.2 $(\mathrm{t}, \mathrm{C} 11)$.

IR (KBr): 3398, 3070, 2926, 2893, 2865, 2846, 1640, 1457, 1437, 1021, 1000, $879 \mathrm{~cm}^{-1}$.

EI-MS m/z (\%): $220\left(\mathrm{M}^{+}, 30\right), 205$ (14), 202 (11), 189 (100), 177 (15), 159 (38), 152 (16), 147 (24), 133 (53), 119 (35), 107 (51), 91 (51), 79 (32), 67 (19), 55 (21), 41 (22).

HR-EI-MS Calcd for $\mathrm{C}_{15} \mathrm{H}_{24} \mathrm{O}: \mathrm{m} / z$ 220.1827. Found: 220.1824. 


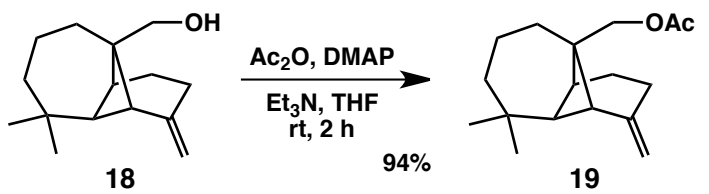

To a solution of $18(72.3 \mathrm{mg}, 0.328 \mathrm{mmol})$ in THF $(3.3 \mathrm{~mL})$ were added $\mathrm{Et}_{3} \mathrm{~N}(0.28 \mathrm{~mL}, 202 \mathrm{mg}, 2.0 \mathrm{mmol}), \mathrm{Ac}_{2} \mathrm{O}$ $(0.280 .12 \mathrm{~mL}, 133 \mathrm{mg}, 2.0 \mathrm{mmol})$ and DMAP $(4.0 \mathrm{mg}, 0.33 \mathrm{mmol})$ at toom temperature. After being stirred for $2 \mathrm{~h}$, the reaction was quenched by addition of saturated aqueous $\mathrm{NaHCO}_{3}(10 \mathrm{~mL})$ and hexane $(100 \mathrm{~mL})$. The organic layer was washed with saturated aqueous $\mathrm{NaHCO}_{3}$, saturated aqueous $\mathrm{NH}_{4} \mathrm{Cl}$ and brine, dried over $\mathrm{Na}_{2} \mathrm{SO}_{4}$, and concentrated in vacuo. The crude product was purified by column chromatography on silica gel (hexane: $\mathrm{Et}_{2} \mathrm{O}=40: 1$ ) to give 19 as a white solid (80.7 $\mathrm{mg}, 94 \%$ yield).

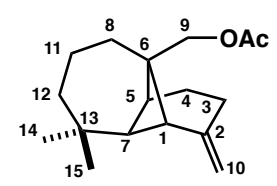

19

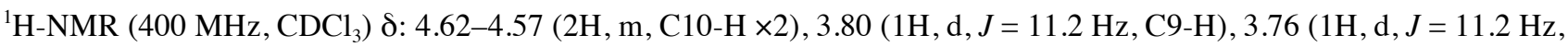
C9-H), 2.67 (1H, d, J = 5.5 Hz, C1-H), 2.62 (1H, m, C3-H), 2.34 (1H, m, C3-H), 2.23 (1H, m, C5-H), 2.02 (3H, s, Ac), 1.96-1.82 (2H, m, C4-H ×2), 1.75-1.54 (4H, m, C8-H ×2, C11-H ×2), $1.56(1 \mathrm{H}, \mathrm{s}, \mathrm{C} 7-\mathrm{H}), 1.46-1.38(2 \mathrm{H}, \mathrm{m}, \mathrm{C} 12-\mathrm{H} \times 2)$, $0.90(6 \mathrm{H}, \mathrm{s}, \mathrm{C} 14 \mathrm{Me}, \mathrm{C} 15 \mathrm{Me})$.

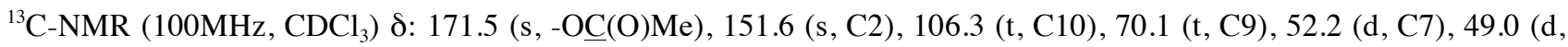
C1), 43.7 (s, C6), 39.5 (t, C12), 37.7 (d, C5), 35.8 (t, C8), 32.6 (s, C13), 28.2 (q, C14 or C15), 27.8 (q, C14 or C15), 25.2 (t, C4), 23.7 (t, C3), 21.1 (t, C11), 20.9 (q, -OC(O)Me).

IR (KBr): 2948, 2926, 2898, 2864, 2849, 1734, 1641, 1461, 1382, 1364, 1245, 1189, 1161, 1030, $903,876 \mathrm{~cm}^{-1}$. EI-MS m/z (\%): 262 (M+, 7.4), 220 (13), 202 (100), 187 (75), 173 (19), 159 (96), 145 (37), 133 (54), 119 (33), 105 (41), 91 (45), 79 (22), 69 (13), 55 (14), 43 (34).

HR-EI-MS Calcd for $\mathrm{C}_{17} \mathrm{H}_{26} \mathrm{O}_{2}: m / z, 262.1933$. Found: 262.1934.

\section{9-Acetoxy-3-epi-marsupellol}

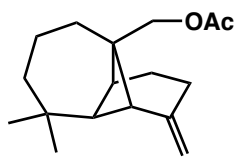

19

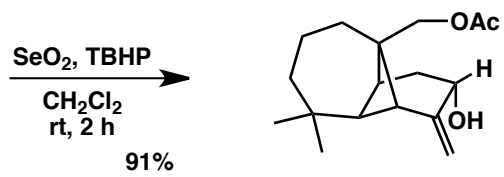

9-acetoxy-3-epi-marsupellol

To the solution of $19(80.7 \mathrm{mg}, 0.308 \mathrm{mmol})$ in $\mathrm{CH}_{2} \mathrm{Cl}_{2}(3.1 \mathrm{~mL})$ were added TBHP in nonane $(5.5 \mathrm{M}, 0.11 \mathrm{~mL}, 0.62$ mmol $)$ and $\mathrm{SeO}_{2}(3.4 \mathrm{mg}, 0.031 \mathrm{mmol})$ at room temperature and the mixture was stirred for $2 \mathrm{~h}$. The reaction was quenched by addition of saturated aqueous $\mathrm{NaHCO}_{3}(5 \mathrm{~mL}), 20 \%$ aqueous $\mathrm{Na}_{2} \mathrm{~S}_{2} \mathrm{O}_{3}(5 \mathrm{~mL})$, and $\mathrm{Et}_{2} \mathrm{O}(30 \mathrm{~mL})$. The aqueous layer was separated and extracted with $\mathrm{Et}_{2} \mathrm{O}(30 \mathrm{~mL})$. The combined organic layers were washed brine, dried over $\mathrm{Na}_{2} \mathrm{SO}_{4}$, and concentrated in vacuo. The crude product was purified by column chromatography on silica gel (hexane:AcOEt = 10:1-4:1) to give 9-Acetoxy-3-epi-marsupellol as a white solid (78.2 mg, 91\% yield). 


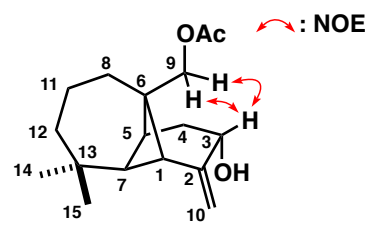

9-acetoxy-3-epi-marsupellol

${ }^{1} \mathrm{H}-\mathrm{NMR}\left(400 \mathrm{MHz}, \mathrm{CDCl}_{3}\right)$ d: $5.00(1 \mathrm{H}, \mathrm{t}, J=0.9 \mathrm{~Hz}, \mathrm{C} 10-\mathrm{H}), 4.87(1 \mathrm{H}, \mathrm{m}, \mathrm{C} 10-\mathrm{H}), 4.53(1 \mathrm{H}, \mathrm{d}, J=8.0 \mathrm{~Hz}, \mathrm{C} 3-\mathrm{H})$, $3.63(1 \mathrm{H}, \mathrm{d}, J=11.4 \mathrm{~Hz}, \mathrm{C} 9-\mathrm{H}), 3.58(1 \mathrm{H}, \mathrm{d}, J=11.4 \mathrm{~Hz}, \mathrm{C} 9-\mathrm{H}), 2.74(1 \mathrm{H}, \mathrm{d}, J=5.5 \mathrm{~Hz}, \mathrm{C} 1-\mathrm{H}), 2.33(1 \mathrm{H}, \mathrm{ddd}, J=$ 14.6, 8.0, $2.1 \mathrm{~Hz}, \mathrm{C} 4-\mathrm{Hb}), 2.27(1 \mathrm{H}, \mathrm{m}, \mathrm{C} 5-\mathrm{H}), 2.02(3 \mathrm{H}, \mathrm{s}, \mathrm{Ac}), 1.90(1 \mathrm{H}, \mathrm{s}, \mathrm{C} 7-\mathrm{H}), 1.89(1 \mathrm{H}, \mathrm{dd}, J=14.6,3.8 \mathrm{~Hz}$, C4-Ha), 1.73 (1H, m, C8-H), 1.68-1.59 (3H, m, C11-H ×2, C8-H), 1.46-1.40 (2H, m, C12-H ×2), 0.93 (3H, s, C14 Me or $\mathrm{C} 15 \mathrm{Me}), 0.92$ (3H, s, C14 Me or C15 Me).

The NOEs shown in the figure were observed.

${ }^{13} \mathrm{C}-\mathrm{NMR}\left(100 \mathrm{MHz}, \mathrm{CDCl}_{3}\right.$ ) d: 171.4 (s, -OC(O)Me), 156.4 (s, C2), 111.2 (t, C10), 69.2 (t, C9), 67.1 (d, C3), 52.7 (d, C7), 48.0 (d, C1), 43.4 (s, C6), 39.5 (t, C12), 37.3 (d, C5), 36.7 (t, C4), 35.7 (t, C8), 32.6 (s, C13), 28.1 (q, C14 or C15), 27.6 (q, C14 or C15), 21.2 (t, C11), 20.9 (q, -OC(O)Me).

IR (KBr): 3399, 3356, 2929, 2948, 2897, 2866, 2847, 1737, 1469, 1455, 1387, 1362, 1255, 1244, 1227, 1030, 1012, 972 , $905 \mathrm{~cm}^{-1}$.

HR-ESI-MS Calcd for $\mathrm{C}_{17} \mathrm{H}_{26} \mathrm{NaO}_{3}: \mathrm{m} / z$ 301.1780. Found: $301.1783\left(\mathrm{M}+\mathrm{Na}^{+}\right)$.

\section{Acetoxymarsupellone}
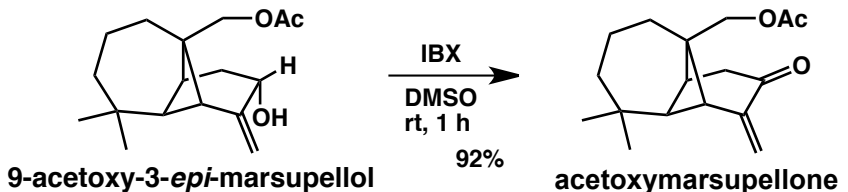

To a solution of 9-acetoxy-3-epi-marsupellol $(67.7 \mathrm{mg}, 0.241 \mathrm{mmol})$ in DMSO $(2.4 \mathrm{~mL})$ was added IBX (135 mg, $0.483 \mathrm{mmol})$ at room temperature. After being stirred for $1 \mathrm{~h}$, hexane: $\mathrm{Et}_{2} \mathrm{O}(=1: 10,30 \mathrm{~mL})$ and water $(5 \mathrm{~mL})$ were added, and the resulting precipitate was removed by filtration through celite. The filtered organic layer was separated, washed with saturated aqueous $\mathrm{NaHCO}_{3}$ and brine, dried over $\mathrm{Na}_{2} \mathrm{SO}_{4}$, and concentrated in vacuo. The crude product was purified by column chromatography on silica gel (hexane:AcOEt $=10: 1$ ) to give acetoxymarsupellone as a colorless oil (61.7 mg, 92\% yield).

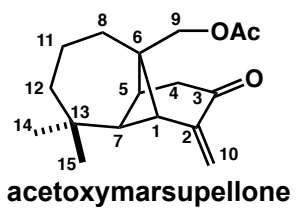

${ }^{1} \mathrm{H}-\mathrm{NMR}\left(400 \mathrm{MHz}, \mathrm{CDCl}_{3}\right)$ d: $5.93(1 \mathrm{H}, \mathrm{d}, J=1.2 \mathrm{~Hz}, \mathrm{C} 10-\mathrm{H}), 5.03(1 \mathrm{H}, \mathrm{d}, J=1.2 \mathrm{~Hz}, \mathrm{C} 10-\mathrm{H}), 3.79(1 \mathrm{H}, \mathrm{d}, J=11.8$ Hz, C9-H), 3.75 (1H, d, J = 11.8 Hz, C9-H), 2.96 (1H, d, J = 6.9 Hz, C1-H), 2.74 (1H, dd, J = 19.2, 2.5 Hz, C4-Hb), $2.57(1 \mathrm{H}, \mathrm{dd}, J=19.2,3.4 \mathrm{~Hz}, \mathrm{C} 4-\mathrm{Ha}), 2.46(1 \mathrm{H}, \mathrm{m}, \mathrm{C} 5-\mathrm{H}), 2.01(3 \mathrm{H}, \mathrm{s}, \mathrm{Ac}), 1.81(1 \mathrm{H}, \mathrm{m}, \mathrm{C} 8-\mathrm{H}), 1.74-1.65(3 \mathrm{H}, \mathrm{m}$, C11-H ×2, C8-H), 1.51-1.45 (2H, m, C12-H ×2), 1.42 (1H, s, C7-H), 0.94 (3H, s, C14 Me or C15 Me), 0.94 (3H, s, C14 Me or C15 Me).

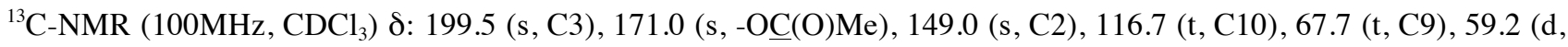
C7), 45.1 (d, C1), 44.2 (t, C4), 43.9 (s, C6), 39.4 (t, C12), 35.72 (d, C5), 35.68 (t, C8), 33.1 (s, C13), 27.8 (q, C14 or 
C15), 27.3 (q, C14 or C15), 20.9 (t, C11), 20.7 (q, -OC(O) $\underline{\mathrm{Me}})$.

IR (neat): 2949, 2932, 2905, 2868, 1740, 1707, 1626, 1469, 1456, 1385, 1363, 1281, 1237, 1102, 1037, $934 \mathrm{~cm}^{-1}$.

EI-MS m/z (\%): $276\left(\mathrm{M}^{+}, 20\right), 234$ (40), 216 (100), 201 (52), 188 (27), 173 (94), 160 (37), 147 (53), 133 (39), 120 (56), 107

(32), 91 (46), 79 (35), 67 (23), 55 (23), 43 (69).

HR-EI-MS Calcd for $\mathrm{C}_{17} \mathrm{H}_{24} \mathrm{O}_{3}: m / z$ 276.1725. Found: 276.1726.

\section{9-Acetoxymarsupellol}

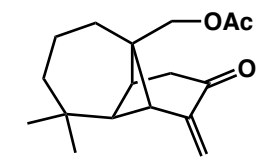

acetoxymarsupellone

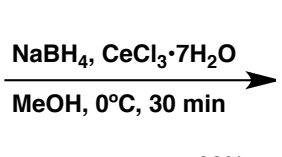

$90 \%$

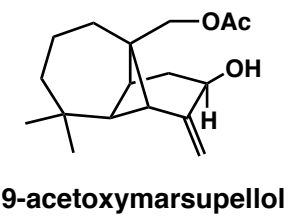

9-acetoxymarsupellol

To the solution of acetoxymarsupellone $(12.2 \mathrm{mg}, 0.0438 \mathrm{mmol})$ in $\mathrm{MeOH}(0.4 \mathrm{~mL})$ was added a suspension of $\mathrm{NaBH}_{4}$ $(8.3 \mathrm{mg}, 0.22 \mathrm{mmol})$ and $\mathrm{CeCl}_{3} \cdot 7 \mathrm{H}_{2} \mathrm{O}(81.6 \mathrm{mg}, 0.219 \mathrm{mmol})$ in $\mathrm{MeOH}(0.4 \mathrm{~mL})$ at $0{ }^{\circ} \mathrm{C}$ and the mixture was stirred for $30 \mathrm{~min}$. An aqueous $\mathrm{NH}_{4} \mathrm{Cl}(3 \mathrm{~mL})$ was added and the mixture was concentrated in vacuo to a half volume. The reaction mixture was diluted with $\mathrm{Et}_{2} \mathrm{O}$, aqueous $\mathrm{HCl}(1.0 \mathrm{M})$ was added and the organic layer was washed with brine and dried over $\mathrm{Na}_{2} \mathrm{SO}_{4}$, and concentrated in vacuo. The crude product was purified by column chromatography on silica gel (hexane:AcOEt = 4:1) to give 9-acetoxymarsupellol as a white solid (11.0 mg, 90\% yield).

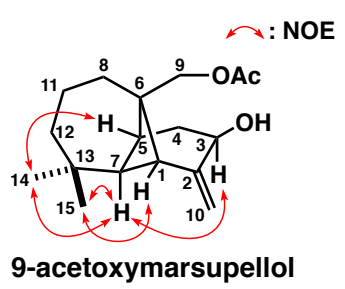

${ }^{1} \mathrm{H}-\mathrm{NMR}\left(400 \mathrm{MHz}, \mathrm{CDCl}_{3}\right)$ d: $5.05(1 \mathrm{H}, \mathrm{dd}, J=2.4,0.9 \mathrm{~Hz}, \mathrm{C} 10-\mathrm{H}), 4.85(1 \mathrm{H}, \mathrm{d}, J=2.4 \mathrm{~Hz}, \mathrm{C} 10-\mathrm{H}), 4.70(1 \mathrm{H}, \mathrm{m}$, C3-H), $3.88(1 \mathrm{H}, \mathrm{d}, J=11.4 \mathrm{~Hz}, \mathrm{C} 9-\mathrm{H}), 3.85$ (1H, d, $J=11.4 \mathrm{~Hz}, \mathrm{C} 9-\mathrm{H}), 2.80(1 \mathrm{H}, \mathrm{d}, J=6.2 \mathrm{~Hz}, \mathrm{C} 1-\mathrm{H}), 2.54(1 \mathrm{H}, \mathrm{ddd}$, $J=14.0,9.8,4.4 \mathrm{~Hz}, \mathrm{C} 4-\mathrm{H} \alpha), 2.30(1 \mathrm{H}, \mathrm{m}, \mathrm{C} 5-\mathrm{H}), 2.02(3 \mathrm{H}, \mathrm{s}, \mathrm{Ac}), 1.77$ (1H, ddd, $J=14.0,6.2,1.8 \mathrm{~Hz}, \mathrm{C} 4-\mathrm{H} \beta), 1.77-$ $1.68(1 \mathrm{H}, \mathrm{m}, \mathrm{C} 8-\mathrm{H}), 1.67-1.57(3 \mathrm{H}, \mathrm{m}, \mathrm{C} 11-\mathrm{H} \times 2, \mathrm{C} 8-\mathrm{H}), 1.52(1 \mathrm{H}, \mathrm{s}, \mathrm{C} 7-\mathrm{H}), 1.45-1.39(2 \mathrm{H}, \mathrm{m}, \mathrm{C} 12-\mathrm{H} \times 2), 0.90(3 \mathrm{H}, \mathrm{s}$, C14 Me), 0.89 (3H, s, C15 Me).

The NOEs shown in the figure were observed.

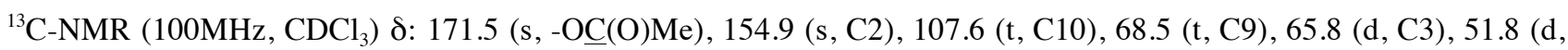
C7), 48.4 (d, C1), 44.6 (s, C6), 39.3 (t, C13), 38.0 (d, C5), 37.0 (t, C4), 35.8 (t, C8), 32.7 (s, C13), 28.3 (q, C15), 27.7 (q, C14), 21.0 (t, C11), 20.9 (q, -OC(O) $\underline{\mathrm{Me}})$.

IR (KBr): 3471, 2930, 2867, 1739, 1456, 1387, 1364, 1241, 1033, 893, $738 \mathrm{~cm}^{-1}$.

HR-ESI-MS Calcd for $\mathrm{C}_{17} \mathrm{H}_{26} \mathrm{NaO}_{3}: m / z$ 301.1780. Found: $301.1777\left(\mathrm{M}+\mathrm{Na}^{+}\right)$. 


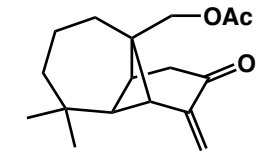

acetoxymarsupellone

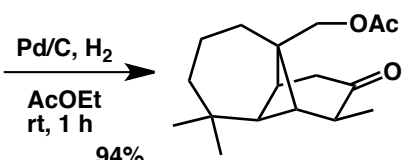

20

To a solution of acetoxymarsupellone $(38.2 \mathrm{mg}, 0.137 \mathrm{mmol})$ in AcOEt $(1.4 \mathrm{~mL})$ was added Pd/C $(10 \%, 14.6 \mathrm{mg}$, $0.0137 \mathrm{mmol}$ ) at room temperature and the mixture was stirred for $1 \mathrm{~h}$ under hydrogen (balloon). AcOEt (20 mL) was added to the mixture. The precipitate was removed by filtration through celite, and the filtrate was concentrated in vacuo. The crude product was purified by column chromatography on silica gel (hexane:AcOEt $=10: 1$ ) to give $\mathbf{2 0}$ as a colorless oil (36.3 mg, $94 \%$ yield).

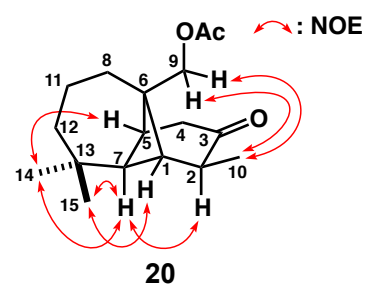

${ }^{1} \mathrm{H}-\mathrm{NMR}\left(400 \mathrm{MHz}, \mathrm{CDCl}_{3}\right)$ \&: $3.86(1 \mathrm{H}, \mathrm{d}, J=11.7 \mathrm{~Hz}, \mathrm{C} 9-\mathrm{H}), 3.80(1 \mathrm{H}, \mathrm{d}, J=11.7 \mathrm{~Hz}, \mathrm{C} 9-\mathrm{H}), 2.65(1 \mathrm{H}, \mathrm{dd}, J=19.0$, $2.9 \mathrm{~Hz}, \mathrm{C} 4-\mathrm{H}), 2.54(1 \mathrm{H}, \mathrm{dd}, J=19.0,2.9 \mathrm{~Hz}, \mathrm{C} 4-\mathrm{H}), 2.48(1 \mathrm{H}, \mathrm{m}, \mathrm{C} 2-\mathrm{H}), 2.37$ (1H, dt, $J=6.1,2.9, \mathrm{C} 5-\mathrm{H}), 2.27(1 \mathrm{H}, \mathrm{m}$, C1-H), 2.03 (3H, s, Ac), 1.80-1.60 (4H, m, C8-H ×2, C11-H ×2), 1.47-1.41 (2H, m, C12-H ×2), 1.33 (1H, s, C7-H), $1.19(3 \mathrm{H}, \mathrm{d}, J=7.3 \mathrm{~Hz}, \mathrm{C} 10 \mathrm{Me}), 0.95(3 \mathrm{H}, \mathrm{s}, \mathrm{C} 15 \mathrm{Me}), 0.94(3 \mathrm{H}, \mathrm{s}, \mathrm{C} 14 \mathrm{Me})$.

The NOEs shown in the figure were observed.

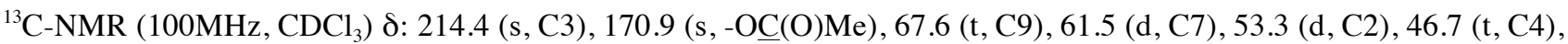
42.6 (d, C1), 42.1 (s, C6), 39.2 (t, C12), 35.96 (t, C8), 35.93 (d, C5), 32.8 (s, C13), 27.9 (q, C14 or C15), 27.6 (q, C14 or C15), 20.9 (t, C11), 20.8 (q, -OC(O)Me), 16.6 (q, C10).

IR (neat): 2933, 2904, 2867, 1743, 1712, 1467, 1456, 1415, 1386, 1363, 1317, 1239, 1123, 1035, $972,907 \mathrm{~cm}^{-1}$.

EI-MS m/z (\%): 278 (M+14), 236 (100), 218 (41), 193 (18), 175 (24), 162 (31), 147 (27), 133 (34), 121 (29), 107 (39), 93 (30), 81 (27), 69 (22), 55 (21), 43 (43).

HR-EI-MS Calcd for $\mathrm{C}_{17} \mathrm{H}_{26} \mathrm{O}_{3}: \mathrm{m} / z$ 278.1882. Found: 278.1881 .

\section{Marsupellin B}

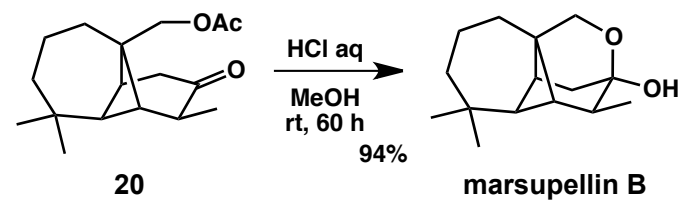

To the solution of $\mathbf{2 0}(32.3 \mathrm{mg}, 0.115 \mathrm{mmol})$ in $\mathrm{MeOH}(1.2 \mathrm{~mL})$ was added aqueous $\mathrm{HCl}(1.0 \mathrm{M}, 1.2 \mathrm{~mL})$. The mixture was stirred at room temperature for $60 \mathrm{~h}$, and concentrated in vacuo to a quarter volume. The residual mixture was diluted with $\mathrm{Et}_{2} \mathrm{O}$, washed with brine, dried over $\mathrm{Na}_{2} \mathrm{SO}_{4}$, and concentrated in vacuo to give marsupellin $\mathrm{B}$ as a white solid (25.6 mg, $94 \%$ yield). 


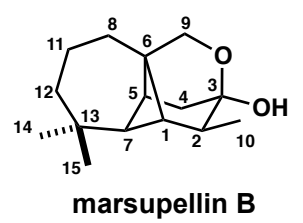

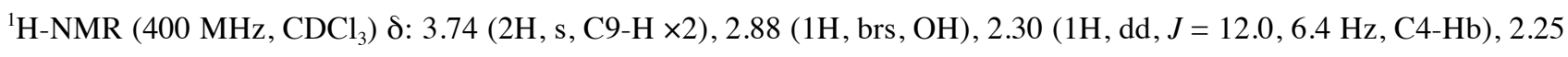
$(1 \mathrm{H}, \mathrm{t}, J=6.4 \mathrm{~Hz}, \mathrm{C} 5-\mathrm{H}), 1.94(1 \mathrm{H}, \mathrm{q}, J=7.0 \mathrm{~Hz}, \mathrm{C} 2-\mathrm{H}), 1.91(1 \mathrm{H}, \mathrm{d}, J=6.4 \mathrm{~Hz}, \mathrm{C} 1-\mathrm{H}), 1.88(1 \mathrm{H}, \mathrm{d}, J=12.0 \mathrm{~Hz}$, C4-Ha), 1.61-1.53 (2H, m, C11-H ×2), 1.42-1.36 (2H, m, C12-H ×2), 1.28-1.22 (2H, m, C8-H ×2), $1.26(1 \mathrm{H}, \mathrm{s}, \mathrm{C} 7-\mathrm{H})$, $1.08(3 \mathrm{H}, \mathrm{d}, J=7.0 \mathrm{~Hz}, \mathrm{C} 10 \mathrm{Me}), 0.90(3 \mathrm{H}, \mathrm{s}, \mathrm{C} 14 \mathrm{Me}$ or C15 Me), $0.89(3 \mathrm{H}, \mathrm{s}, \mathrm{C} 14 \mathrm{Me}$ or C15 Me).

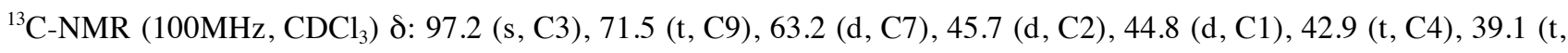
C12), 38.5 (s, C6), 38.3 (d, C5), 32.8 (t, C8), 32.7 (s, C13), 27.8 (q, C14 or C15), 27.7 (q, C14 or C15), 20.9 (t, C11), 14.8 (q, C10).

IR (KBr): 3364, 2956, 2933, 2907, 2861, 2842, 1740, 1718, 1467, 1447, 1361, 1319, 1198, 1112, 1083, 1069 , 1048 , $1026,979,899,879 \mathrm{~cm}^{-1}$.

EI-MS m/z (\%): $236\left(\mathrm{M}^{+}, 100\right), 221$ (12), 207 (10), 193 (43), 180 (12), 176 (19), 163 (19), 152 (11), 149 (15), 133 (22), 123 (36), 107 (63), 95 (29), 81 (39), 69 (27), 55 (22), 41 (20).

HR-EI-MS Calcd for $\mathrm{C}_{15} \mathrm{H}_{24} \mathrm{O}_{2}: \mathrm{m} / z$ 236.1776. Found: 236.1774 .

\section{Marsupellin A}

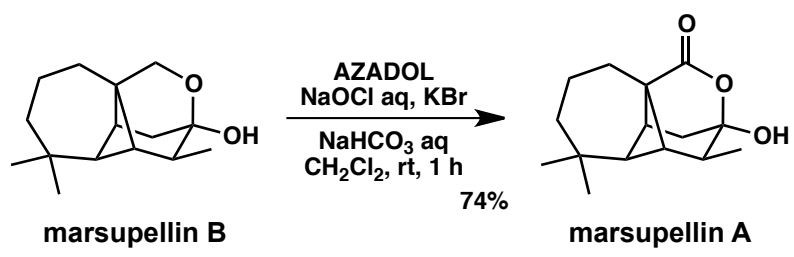

To a two-layers mixture of marsupellin $\mathrm{B}(24.4 \mathrm{mg}, 0.103 \mathrm{mmol})$ in $\mathrm{CH}_{2} \mathrm{Cl}_{2}(1.0 \mathrm{~mL})$ and saturated aqueous $\mathrm{NaHCO}_{3}$ $(1.0 \mathrm{~mL})$ were added $\mathrm{KBr}(1.2 \mathrm{mg}, 10 \mu \mathrm{mol})$, AZADOL in $\mathrm{CH}_{2} \mathrm{Cl}_{2}(0.1 \mathrm{M}, 0.10 \mathrm{~mL}, 1.0 \mu \mathrm{mol})$ and aqueous $\mathrm{NaOCl}$ (ca.1.3 M, $0.40 \mathrm{~mL}, 0.515 \mathrm{~mL}$ ) at room temperature and the mixture was stirred for $1 \mathrm{~h}$. The reaction was quenched by addition of saturated aqueous $\mathrm{NaHCO}_{3}(5 \mathrm{~mL}), 20 \%$ aqueous $\mathrm{Na}_{2} \mathrm{~S}_{2} \mathrm{O}_{3}(5 \mathrm{~mL})$, and $\mathrm{Et}_{2} \mathrm{O}(50 \mathrm{~mL})$. After being stirred for $30 \mathrm{~min}$, the organic layer was separated, washed with brine, dried over $\mathrm{Na}_{2} \mathrm{SO}_{4}$, and concentrated in vacuo. The crude product was purified by column chromatography on silica gel (hexane:AcOEt $=4: 1-3: 1$ ) to give marsupellin A as a white solid (19.2 $\mathrm{mg}, 74 \%$ yield).

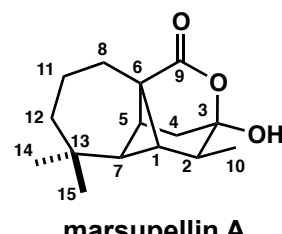

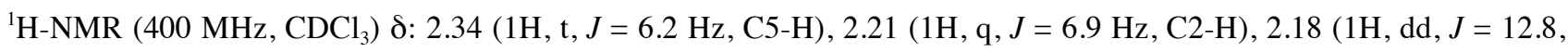
$6.2 \mathrm{~Hz}, \mathrm{C} 4-\mathrm{Hb}), 2.09(1 \mathrm{H}, \mathrm{d}, J=12.8 \mathrm{~Hz}, \mathrm{C} 4-\mathrm{Ha}), 2.03-1.97(2 \mathrm{H}, \mathrm{m}, \mathrm{C} 8-\mathrm{H} \times 2), 1.99(1 \mathrm{H}, \mathrm{d}, J=6.2 \mathrm{~Hz}, \mathrm{C} 1-\mathrm{H}), 1.70-$ $1.62(2 \mathrm{H}, \mathrm{m}, \mathrm{C} 11-\mathrm{H} \times 2), 1.47-1.41(2 \mathrm{H}, \mathrm{m}, \mathrm{C} 12-\mathrm{H} \times 2), 1.32(1 \mathrm{H}, \mathrm{s}, \mathrm{C} 7-\mathrm{H}), 0.96(3 \mathrm{H}, \mathrm{d}, J=6.9 \mathrm{~Hz}, \mathrm{C} 10 \mathrm{Me}), 0.93(6 \mathrm{H}$, $\mathrm{s}, \mathrm{C} 14 \mathrm{Me}, \mathrm{C} 15 \mathrm{Me})$.

${ }^{13} \mathrm{C}-\mathrm{NMR}\left(100 \mathrm{MHz}, \mathrm{CDCl}_{3}\right.$ ) : 174.3 (s, C9), 101.9 (s, C3), 63.2 (d, C7), 45.7 (d, C2), 44.4 (s, C6), 43.7 (d, C1), 43.0 (t, C4), 38.7 (t, C12), 36.4 (d, C5), 32.7 (s, C13), 30.2 (t, C8), 27.5 (q, C14 or C15), 27.4 (q, C14 or C15), 20.2 (t, C11), 
12.7 (q, C10).

IR (KBr): 3381, 2934, 2903, 2867, 1731, 1709, 1456, 1363, 1326, 1293, 1216, 1181, 1142, 1113, 1090, 1068, 1028, 986, $958,934,927 \mathrm{~cm}^{-1}$.

EI-MS m/z (\%): 250 (M+ , 38), 232 (18), 222 (13), 206 (31), 191 (100), 181 (51), 177 (23), 163 (23), 149 (29), 135 (35), 121 (39), 107 (33), 93 (26), 81 (28), 69 (30), 55 (22), 41 (25).

HR-EI-MS Calcd for $\mathrm{C}_{15} \mathrm{H}_{22} \mathrm{O}_{3}: \mathrm{m} / z$ 250.1569. Found: 250.1568. 


\begin{tabular}{|c|c|c|c|c|}
\hline & 9-acetoxy-3-epi-1 & arsupellol & 9-acetoxy & narsupellol \\
\hline & Synthetic compound & Lou $^{6}$ & Synthetic compound & Nagashima and Asakawa $^{7}$ \\
\hline 夏H-NMR & $400 \mathrm{MHz}$ & $600 \mathrm{MHz}$ & $400 \mathrm{MHz}$ & $400 \mathrm{MHz}$ \\
\hline position & & & & \\
\hline 1 & $2.74 \mathrm{~d}(5.5)$ & $2.74 \mathrm{~d}(5.4)$ & $2.80 \mathrm{~d}(6.4)$ & $2.80 \mathrm{~d}(6.4)$ \\
\hline 2 & & & & \\
\hline 3 & $4.53 \mathrm{~d}(8.0)$ & $4.54 \mathrm{~d}(7.8)$ & $4.70 \mathrm{dt}(3.2,6.2)$ & $4.70 \mathrm{~m}$ \\
\hline $4 \alpha$ & $1.89 \mathrm{dd}(14.6,3.8)$ & $1.64 \mathrm{~m}$ & $2.54 \mathrm{ddd}(14.0,9.8,4.4)$ & $2.54 \mathrm{ddd}(14.2,9.8,4.4)$ \\
\hline $4 \beta$ & 2.33 ddd $(14.6,8.0,2.2)$ & $2.34 \mathrm{~m}$ & 1.77 ddd $(14.0,6.2,1.8)$ & $1.80-1.69 \mathrm{~m}$ \\
\hline 5 & $2.27 \mathrm{~m}$ & $2.27 \mathrm{~m}$ & $2.30 \mathrm{~m}$ & $2.30 \mathrm{brt}(5.4)$ \\
\hline 6 & & & & \\
\hline 7 & $1.90 \mathrm{~s}$ & $1.90 \mathrm{~s}$ & $1.52 \mathrm{~s}$ & $1.52 \mathrm{~s}$ \\
\hline 8 & $1.73 \mathrm{~m}$ & $1.75 \mathrm{~m}$ & $1.77-1.68 \mathrm{~m}$ & $1.80-1.69 \mathrm{~m}$ \\
\hline & $1.68-1.59 \mathrm{~m}$ & $1.64 \mathrm{~m}$ & $1.67-1.57 \mathrm{~m}$ & $1.66-1.57 \mathrm{~m}$ \\
\hline 9 & $3.63 \mathrm{~d}(11.4)$ & $3.63 \mathrm{~d}(11.4)$ & $3.88 \mathrm{~d}(11.4)$ & $3.86 \mathrm{~s}$ \\
\hline & $3.58 \mathrm{~d}(11.4)$ & $3.59 \mathrm{~d}(11.4)$ & $3.85 \mathrm{~d}(11.4)$ & $3.87 \mathrm{~s}$ \\
\hline 10 & $5.00 \mathrm{t}(0.9)$ & $4.99 \mathrm{~s}$ & $5.05 \mathrm{dd}(2.4,0.9)$ & $5.05 \mathrm{~s}$ \\
\hline & $4.87 \mathrm{~m}$ & $4.87 \mathrm{~s}$ & $4.85 \mathrm{~d}(2.4)$ & $4.85 \mathrm{~s}$ \\
\hline 11 & $1.68-1.59 \mathrm{~m}, 2 \mathrm{H}$ & $1.64 \mathrm{~m}, 2 \mathrm{H}$ & $1.67-1.57 \mathrm{~m}, 2 \mathrm{H}$ & $1.66-1.57 \mathrm{~m}, 2 \mathrm{H}$ \\
\hline 12 & $1.46-1.40 \mathrm{~m}, 2 \mathrm{H}$ & $1.44 \mathrm{~m}, 2 \mathrm{H}$ & $1.45-1.39 \mathrm{~m}, 2 \mathrm{H}$ & $1.42 \mathrm{~m}, 2 \mathrm{H}$ \\
\hline 13 & & & & \\
\hline 14 & $0.93 \mathrm{~s}, 3 \mathrm{H}$ & $0.93 \mathrm{~s}, 3 \mathrm{H}$ & $0.90 \mathrm{~s}, 3 \mathrm{H}$ & $0.90 \mathrm{~s}, 3 \mathrm{H}$ \\
\hline 15 & $0.92 \mathrm{~s}, 3 \mathrm{H}$ & $0.92 \mathrm{~s}, 3 \mathrm{H}$ & $0.89 \mathrm{~s}, 3 \mathrm{H}$ & $0.89 \mathrm{~s}, 3 \mathrm{H}$ \\
\hline OAc & $2.02 \mathrm{~s}, 3 \mathrm{H}$ & $2.01 \mathrm{~s}, 3 \mathrm{H}$ & $2.02 \mathrm{~s}, 3 \mathrm{H}$ & $2.02 \mathrm{~s}, 3 \mathrm{H}$ \\
\hline${ }^{13} \mathrm{C}-\mathrm{NMR}$ & $100 \mathrm{MHz}$ & $150 \mathrm{MHz}$ & $100 \mathrm{MHz}$ & $100 \mathrm{MHz}$ \\
\hline position & & & & \\
\hline 1 & 48.0 & 48.1 & 48.4 & 48.4 \\
\hline 2 & 156.4 & 156.6 & 154.9 & 154.9 \\
\hline 3 & 67.1 & 67.3 & 65.8 & 65.8 \\
\hline 4 & 36.7 & 36.8 & 37.0 & 37.0 \\
\hline 5 & 37.3 & 37.5 & 38.0 & 38.0 \\
\hline 6 & 43.4 & 43.5 & 44.6 & 44.6 \\
\hline 7 & 52.7 & 52.8 & 51.8 & 51.8 \\
\hline 8 & 35.7 & 35.8 & 35.8 & 35.8 \\
\hline 9 & 69.2 & 69.4 & 68.5 & 68.5 \\
\hline 10 & 111.2 & 111.4 & 107.6 & 107.6 \\
\hline 11 & 21.2 & 21.0 & 21.0 & 21.1 \\
\hline 12 & 39.5 & 39.7 & 39.3 & 39.3 \\
\hline 13 & 32.6 & 32.8 & 32.7 & 32.8 \\
\hline 14 & 27.6 & 27.7 & 27.7 & 27.8 \\
\hline 15 & 28.1 & 28.3 & 28.3 & 28.3 \\
\hline -OCOMe & 171.4 & 171.6 & 171.5 & 171.5 \\
\hline -OCOMe & 20.9 & 21.3 & 20.9 & 21.0 \\
\hline
\end{tabular}

Although the compound which was isolated from the Chinese liverwort Marsupella alpine by Lou and co-workers was reported as 9-acetoxymarsupellol, ${ }^{6}$ as a result of the above comparison, we have determined that it is 9-acetoxy-3-epi-marsupellol. 


\section{Acetoxymarsupellone}

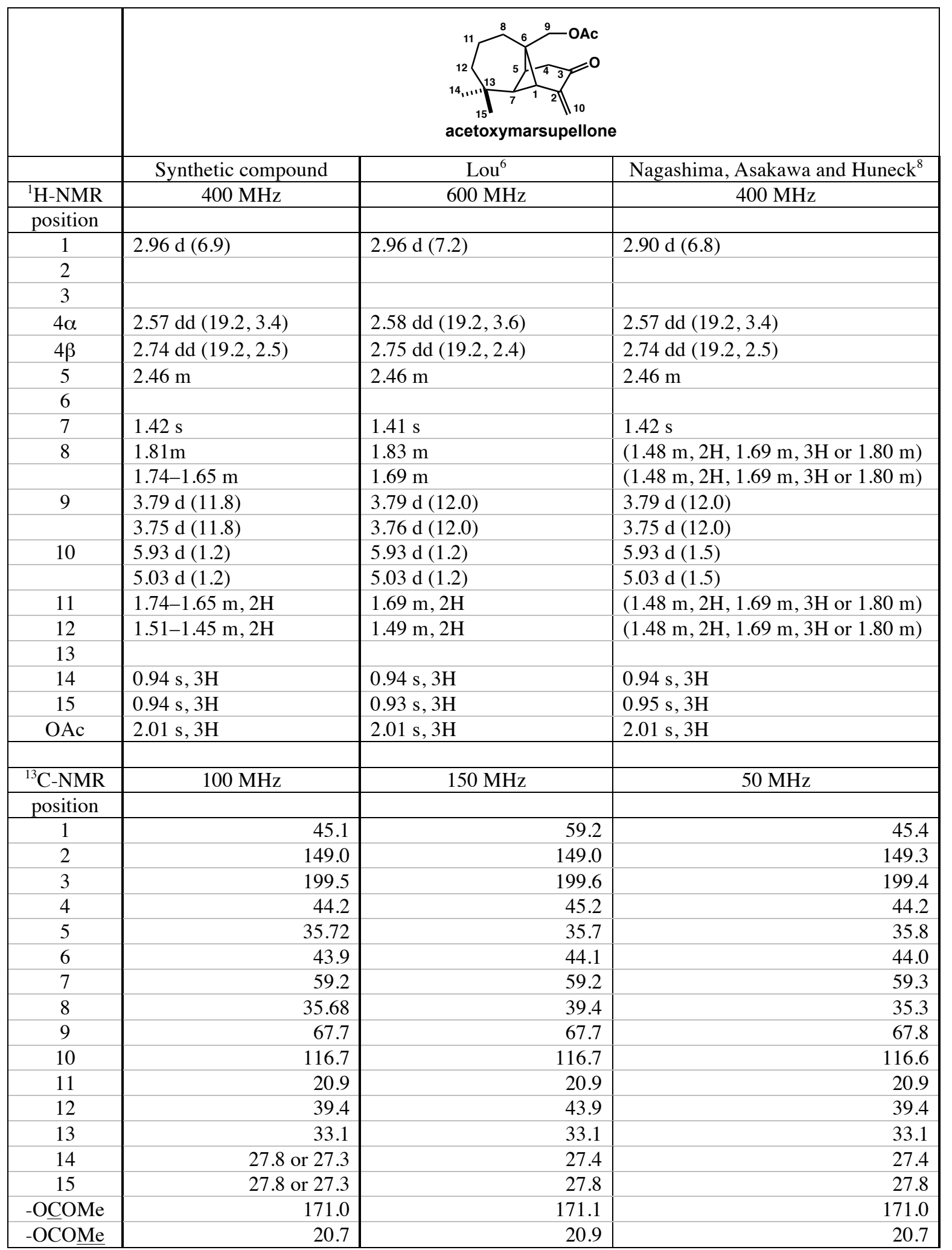

Because details of ${ }^{1} \mathrm{H}-\mathrm{NMR}$ data of acetoxymarsupellone were not described in reference 7 or 9 , data of reference 8 was described. 


\begin{tabular}{|c|c|c|c|c|}
\hline & \multicolumn{2}{|c|}{ marsupellin B } & \multicolumn{2}{|c|}{ marsupellin A } \\
\hline & Synthetic compound & Lou $^{6}$ & Synthetic compound & Lou $^{6}$ \\
\hline${ }^{1} \mathrm{H}-\mathrm{NMR}$ & $400 \mathrm{MHz}$ & $600 \mathrm{MHz}$ & $400 \mathrm{MHz}$ & $600 \mathrm{MHz}$ \\
\hline \multicolumn{5}{|l|}{ position } \\
\hline 1 & $1.91 \mathrm{~d}(6.4)$ & $1.92 \mathrm{~d}(6.0)$ & $1.99 \mathrm{~d}(6.2)$ & $1.99 \mathrm{~m}$ \\
\hline 2 & $1.94 \mathrm{q}(7.0)$ & $1.96 \mathrm{q}(7.0)$ & $2.21 \mathrm{q}(6.9)$ & $2.23 \mathrm{q}(7.2)$ \\
\hline \multicolumn{5}{|l|}{3} \\
\hline $4 \alpha$ & $1.88 \mathrm{~d}(12.0)$ & $1.89 \mathrm{~d}(12.0)$ & $2.09 \mathrm{~d}(12.8)$ & $2.10 \mathrm{~d}(12.6)$ \\
\hline $4 \beta$ & $2.30 \mathrm{dd}(12.0,6.4)$ & $2.30 \mathrm{dd}(12.0,6.0)$ & $2.18 \mathrm{dd}(12.8,6.2)$ & $2.18 \mathrm{dd}(12.6,6.0)$ \\
\hline 5 & $2.25 \mathrm{~d}(6.4)$ & $2.26 \mathrm{~m}$ & $2.34 \mathrm{t}(6.2)$ & $2.34 \mathrm{t}(6.0)$ \\
\hline \multicolumn{5}{|l|}{6} \\
\hline 7 & $1.26 \mathrm{~s}$ & $1.26 \mathrm{~s}$ & $1.32 \mathrm{~s}$ & $1.32 \mathrm{~s}$ \\
\hline 8 & $1.28-1.22 \mathrm{~m}, 2 \mathrm{H}$ & $1.25 \mathrm{~m}, 2 \mathrm{H}$ & $2.03-1.97 \mathrm{~m}, 2 \mathrm{H}$ & $1.99 \mathrm{~m}, 2 \mathrm{H}$ \\
\hline 9 & $3.74 \mathrm{~s}, 2 \mathrm{H}$ & $3.74 \mathrm{~s}, 2 \mathrm{H}$ & & \\
\hline 10 & $1.08 \mathrm{~d}(7.0), 3 \mathrm{H}$ & $1.08 \mathrm{~d}(7.0), 3 \mathrm{H}$ & $0.96 \mathrm{~d}(6.9), 3 \mathrm{H}$ & $0.96 \mathrm{~d}(7.2), 3 \mathrm{H}$ \\
\hline 11 & $1.61-1.53 \mathrm{~m}, 2 \mathrm{H}$ & $1.57 \mathrm{~m}, 2 \mathrm{H}$ & $1.70-1.62 \mathrm{~m}, 2 \mathrm{H}$ & $1.67 \mathrm{~m}, 2 \mathrm{H}$ \\
\hline 12 & $1.42-1.36 \mathrm{~m}, 2 \mathrm{H}$ & $1.39 \mathrm{~m}, 2 \mathrm{H}$ & $1.47-1.41 \mathrm{~m}, 2 \mathrm{H}$ & $1.44 \mathrm{~m}, 2 \mathrm{H}$ \\
\hline \multicolumn{5}{|l|}{13} \\
\hline 14 & $0.90 \mathrm{~s}, 3 \mathrm{H}$ or $0.89 \mathrm{~s}, 3 \mathrm{H}$ & $0.90 \mathrm{~s}, 3 \mathrm{H}$ & $0.93 \mathrm{~s}, 3 \mathrm{H}$ & $0.92 \mathrm{~s}, 3 \mathrm{H}$ \\
\hline 15 & $0.90 \mathrm{~s}, 3 \mathrm{H}$ or $0.89 \mathrm{~s}, 3 \mathrm{H}$ & $0.89 \mathrm{~s}, 3 \mathrm{H}$ & $0.93 \mathrm{~s}, 3 \mathrm{H}$ & $0.92 \mathrm{~s}, 3 \mathrm{H}$ \\
\hline $\mathrm{OH}$ & 2.88 brs & & & \\
\hline${ }^{13} \mathrm{C}-\mathrm{NMR}$ & $100 \mathrm{MHz}$ & $150 \mathrm{MHz}$ & $100 \mathrm{MHz}$ & $150 \mathrm{MHz}$ \\
\hline \multicolumn{5}{|l|}{ position } \\
\hline 1 & 44.8 & 45.0 & 43.7 & 43.8 \\
\hline 2 & 45.7 & 45.8 & 45.7 & 45.8 \\
\hline 3 & 97.2 & 97.3 & 101.9 & 102.2 \\
\hline 4 & 42.9 & 43.0 & 43.0 & 43.1 \\
\hline 5 & 38.3 & 38.5 & 36.4 & 36.5 \\
\hline 6 & 38.5 & 38.7 & 44.4 & 44.5 \\
\hline 7 & 63.2 & 63.4 & 63.2 & 63.3 \\
\hline 8 & 32.8 & 32.9 & 30.2 & 30.3 \\
\hline 9 & 71.5 & 71.7 & 174.3 & 174.7 \\
\hline 10 & 14.8 & 14.9 & 12.7 & 12.9 \\
\hline 11 & 20.9 & 21.0 & 20.2 & 20.3 \\
\hline 12 & 39.1 & 39.3 & 38.7 & $36.8(38.8)^{*}$ \\
\hline 13 & 32.7 & 32.8 & 32.7 & 32.8 \\
\hline 14 & 27.8 or 27.7 & 27.9 & 27.5 or 27.4 & 27.5 \\
\hline 15 & 27.8 or 27.7 & 27.9 & 27.5 or 27.4 & 27.7 \\
\hline
\end{tabular}

* In manuscript, it is described as $36.8 \mathrm{ppm}$, but in ${ }^{13} \mathrm{C}$ NMR spectra of supporting information, there is a signal at 38.8 ppm. 


\section{X-ray Crystallographic Analysis}

X-ray crystallographic data was recorded on a Rigaku R-AXIS RAPID with a MicroMax-007HF diffractometer using multi-layer mirror monochromated $\mathrm{Cu}-\mathrm{Ka}$ radiation. The structure was solved by a direct method (SHELXT ver. 2014/5) ${ }^{10}$ and expanded using a Fourier technique. Refinement was performed using all reflections. The non-hydrogen atoms were refined anisotropically. Hydrogen atoms were refined using the riding model. All calculations were performed using the CrystalStructure 4.2.4 crystallographic software package except for refinement, which was performed using SHELXL ver. 2014/7. ${ }^{11}$

$\left(1 S^{*}, 2 S^{*}, 7 R^{*}, 8 R^{*}, 9 S^{*}\right)$-2-Cyano-8,9-epoxy-6,6-dimethylbicyclo[5.4.0]undec-2-yl methanesulfonate $(7 \alpha)^{12}$

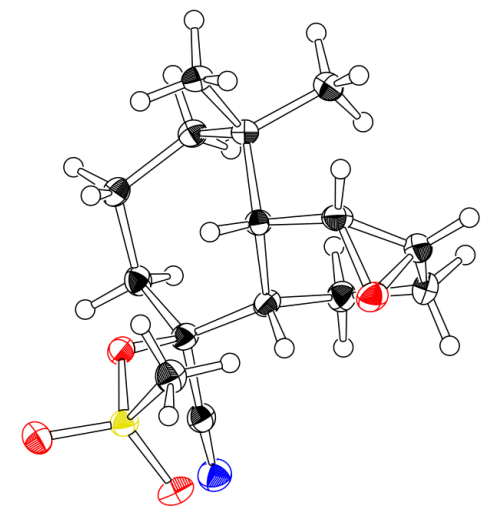

ORTEP drawing of $7 \boldsymbol{\alpha}$

Crystal data for $7 \alpha$

Chemical formula

MW

Crystal system, space group

Temperature $(\mathrm{K})$

$a, b, c(\AA)$

$\alpha, \beta, \gamma\left(\left(^{\circ}\right)\right.$, and $V\left(\AA^{3}\right)$

Z

Radiation type

$\mu\left(\mathrm{mm}^{-1}\right)$

Crystal size (mm), and color

Data collection

Diffractometer

Absorption correction

$T_{\min }, T_{\max }$

No. of measured, independent, and

observed $\left[F^{2}>2.0 \sigma\left(F^{2}\right)\right]$ reflections

$R_{\text {int }}$

$(\sin \theta / \lambda)_{\max }\left(\AA^{-1}\right)$
$\mathrm{C}_{15} \mathrm{H}_{23} \mathrm{NO}_{4} \mathrm{~S}$

313.41

Triclinic, $P_{-1}$

93

7.7561(4), 8.5012(5), $12.0369(7)$

84.266(6), 88.050(6), 70.431(5), 744.08(7)

2

$\mathrm{Cu} K \alpha(\lambda=1.54187 \AA)$

2.08

$0.22 \times 0.16 \times 0.05$ (colorless)

\section{Rigaku R-AXIS RAPID}

Multi-scan

ABSCOR (Rigaku, 1995)

$0.634,0.901$

$8521,2666,2433$

0.048

0.6022

Refinement

$\mathrm{R}\left[F^{2}>2 \sigma\left(F^{2}\right)\right], w R\left(F^{2}\right), S$

$0.042,0.115,1.11$ 
No. of reflections

No. of parameters

$\mathrm{H}$-atom treatment

$D r_{\max }, \mathrm{Dr}_{\text {min }}\left(\mathrm{e} \AA^{-3}\right)$
2666

193

$\mathrm{H}$-atom parameters constrained

$0.39,-0.49$ 


\section{References}

1) Fourgeaud, P.; Midrier, C.; Vors, J. P.; Volle, J. N.; Pirat, J. L.; Virieuxa, D. Tetrahedron 2010, 66, 758-764.

2) (a) Coldham, I.; Jana, S.; Watson, L.; Pilgram, C. D. Tetrahedron Lett. 2008, 49, 5408-5410.

(b) Coldham, I.; Jana, S.; Watson, L.; Martin, N. G. Org. Biomol. Chem. 2009, 7, 1674-1679.

3) Hodgson, D. M.; Chung, Y. K.; Nuzzo, I.; Freixas, G.; Kulikiewicz, K. K.; Cleator, E.; Paris, J. M. J. Am. Chem. Soc. 2007, 129, 4456-4462.

4) (a) K. Matsunaga, K. Takatori, H. Kogen, N. Saito, Tetrahedron Lett. 2018, 59, 3872-3875.

(b) K. Takatori, S. Ota, K. Tendo, K. Matsunaga, K. Nagasawa, S. Watanabe, A. Kishida, H. Kogen, H. Nagaoka, Org. Lett. 2017, 19, 3763-3766.

5) T. C. Stamatatos, S. P. Perlepes, M. J. Manos, A. J. Tasiopoulos, N. Klouras, J. Coord. Chem. 2011, 64, 23772387.

6) Zhang, J.; Fan, P.; Zhu, R.; Li, R.; Lin, Z.; Sun, B.; Zhang, C.; Zhou, J.; Lou, H. J. Nat. Prod. 2014, 77, 10311036.

7) Nagashima, F.; Ishimaru, A.; Asakawa, Y. Phytochemistry 1994, 37, 1767-1768.

8) Nagashima, F.; Ohi, Y.; Nagai, T.; Tori, M.; Asakawa, Y.; Huneck, S. Phytochemistry 1993, 33, $1445-1448$.

9) Matsuo, A.; Uto, S.; Sakuda, K.; Uchino, Y.; Nakayama, M.; Hayashi, S. Chem. Lett. 1979, 73-76.

10) Sheldrick, G. M. Acta Cryst. 2014, A70, C1437.

11) Sheldrick, G. M. Acta Cryst. 2008, A64, 112-122.

12) $\mathrm{CCDC} 1936572$ 


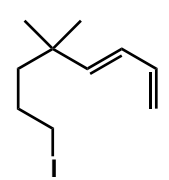

10

10 (1H-NMR)

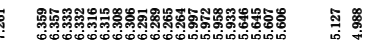
LLI111 YH WH YH

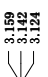

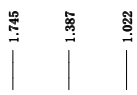

i

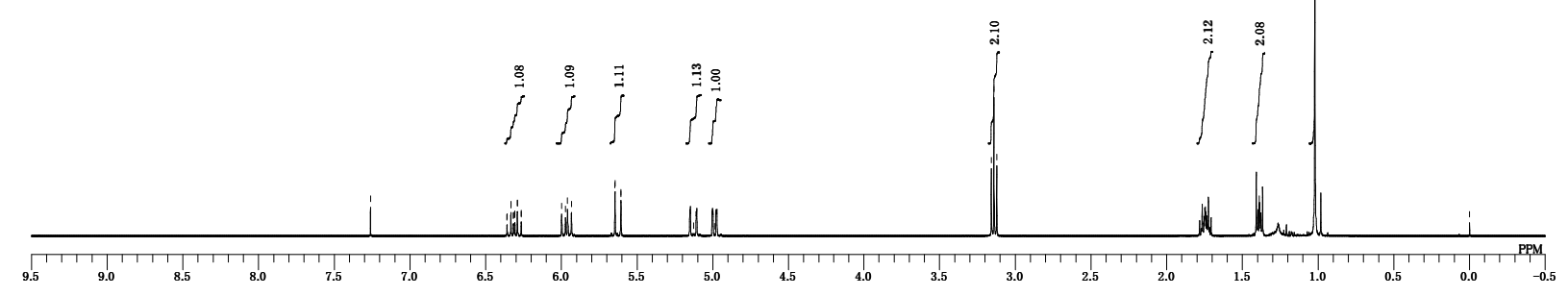

10 (13C-NMR)

$\mid$

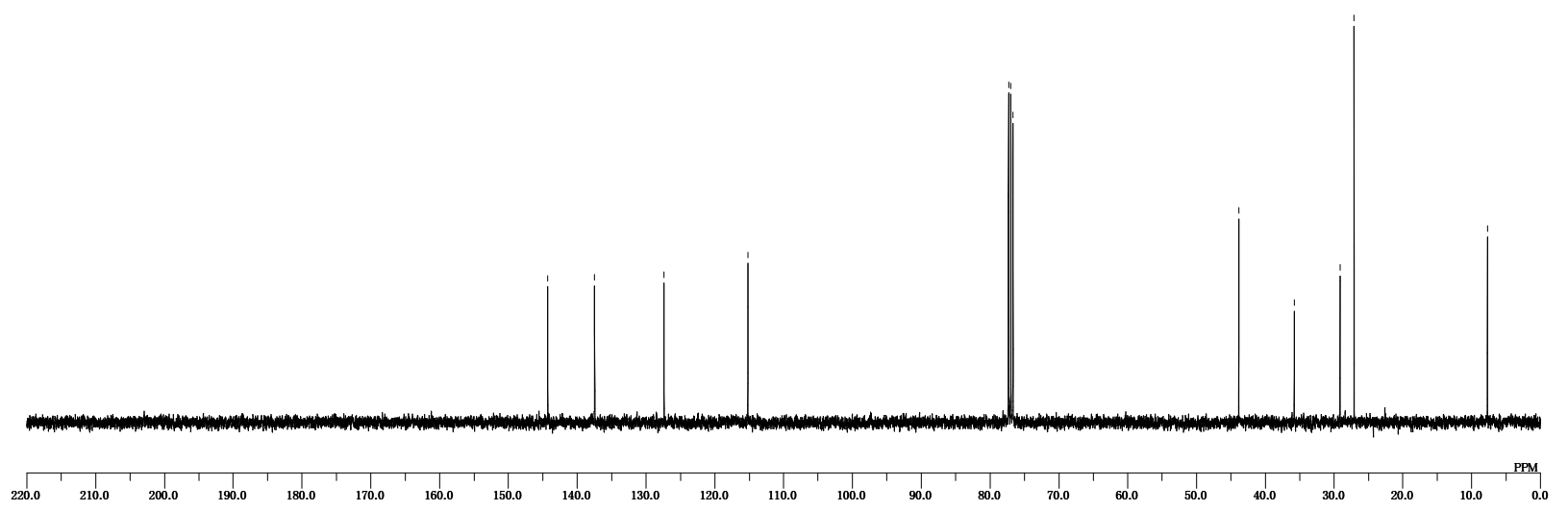




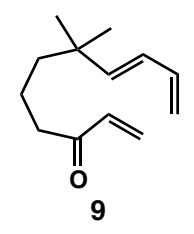

9 (1H-NMR)

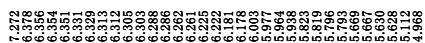

$11111111111111 \mid 111111111)$

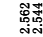

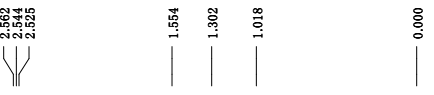

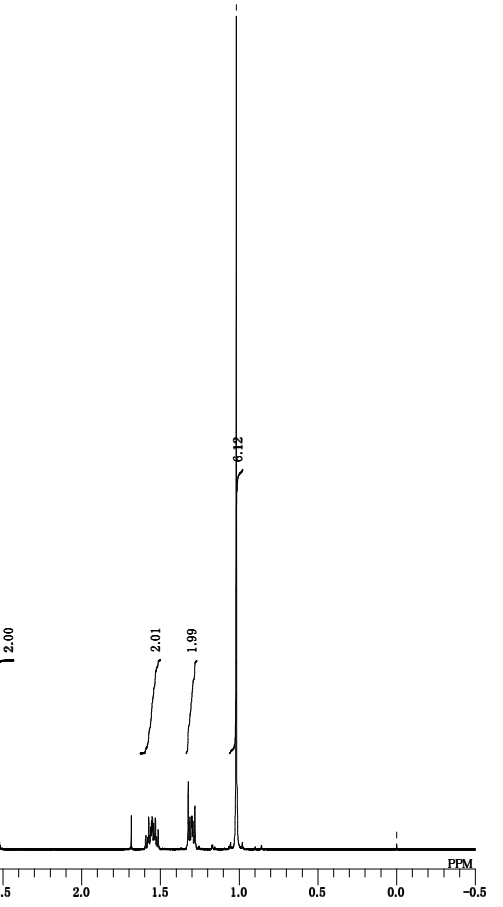

9 (13C-NMR)

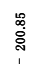

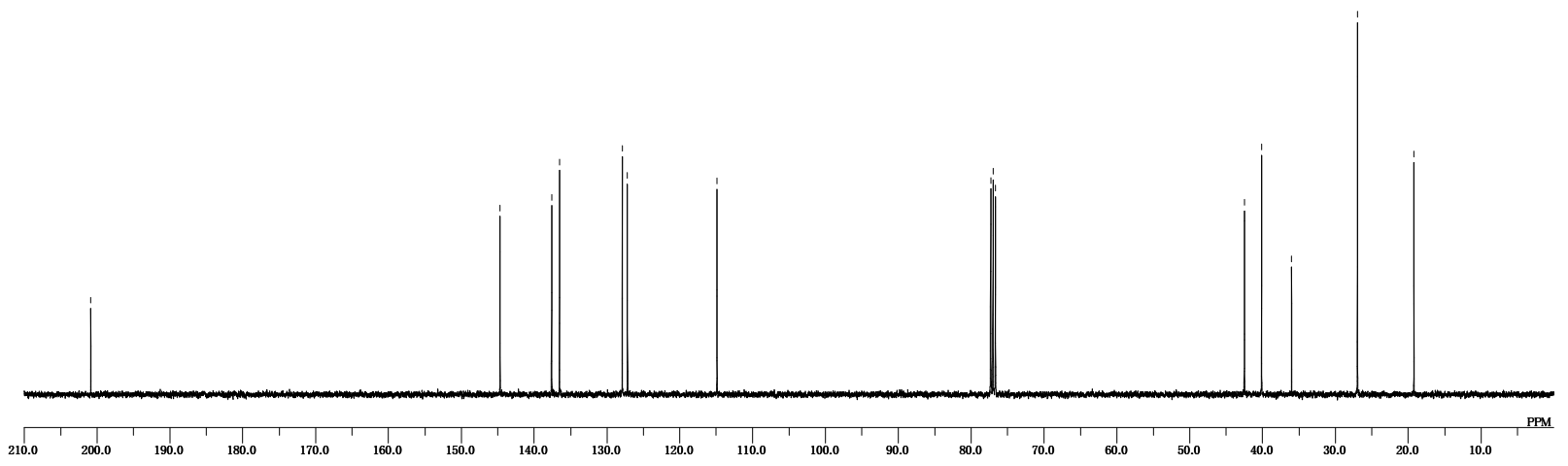




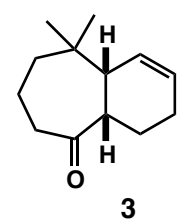

3 (1H-NMR)

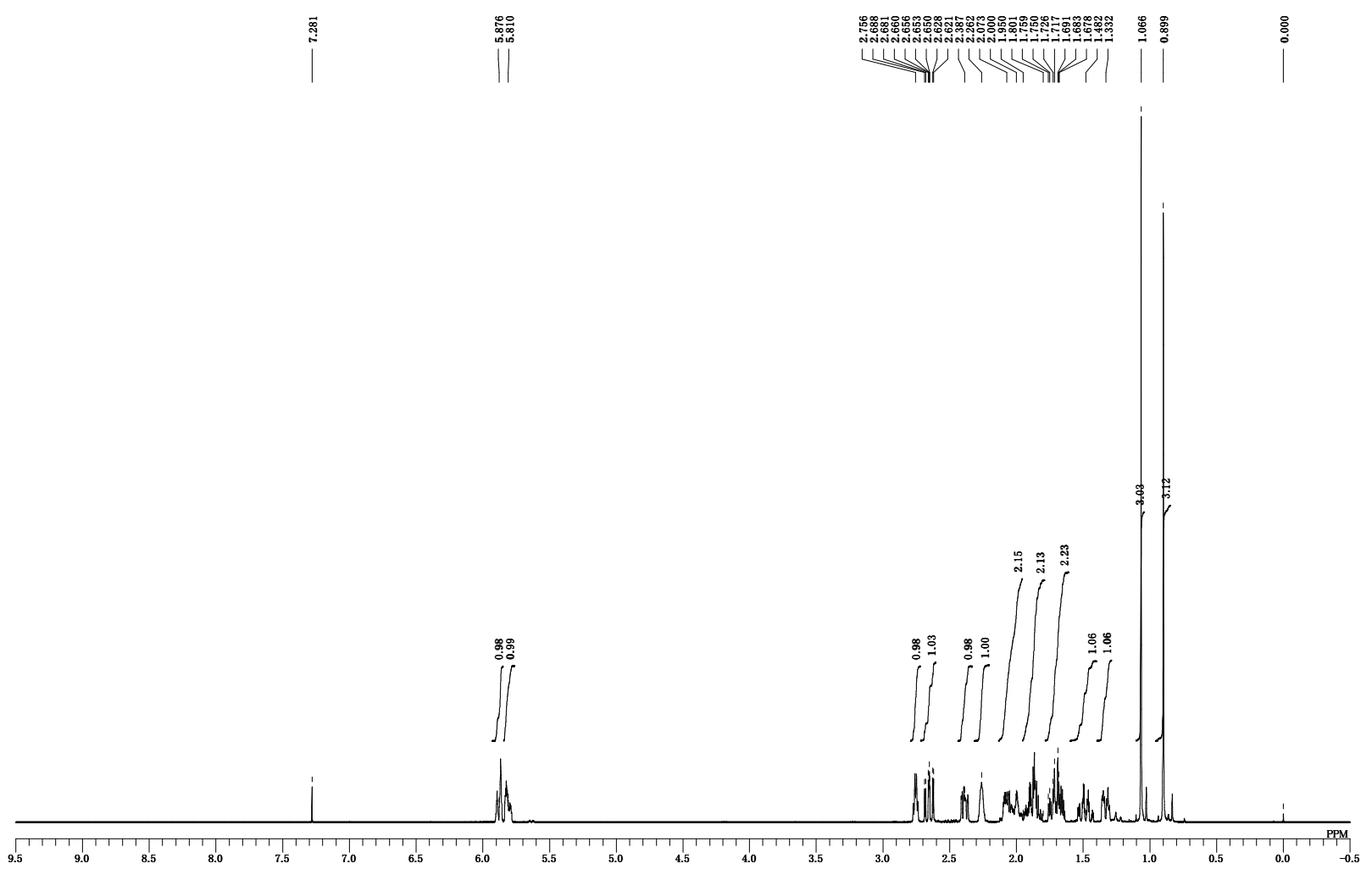

3 (13C-NMR)

|

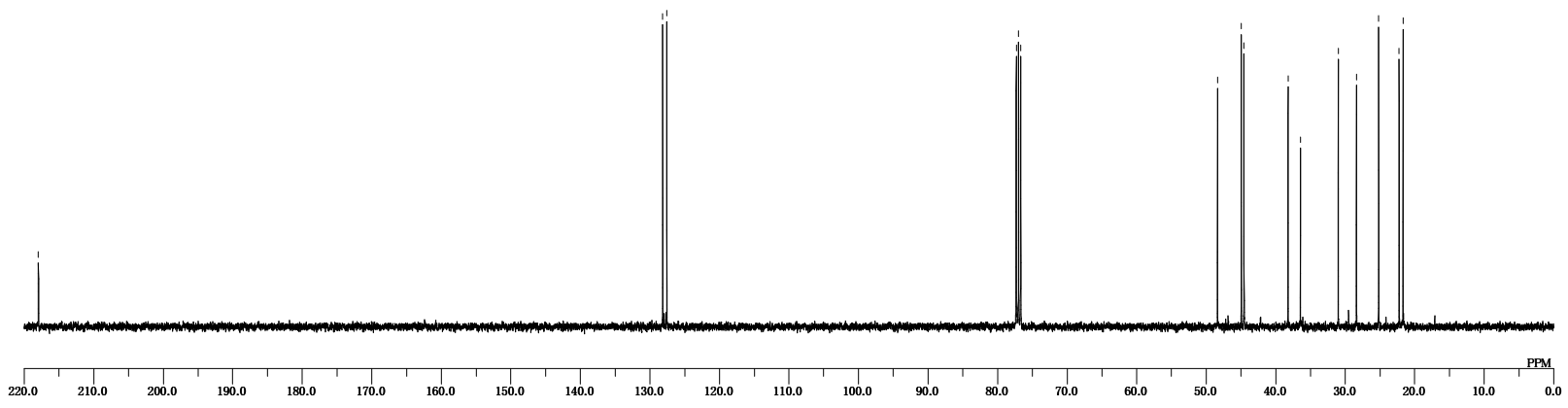



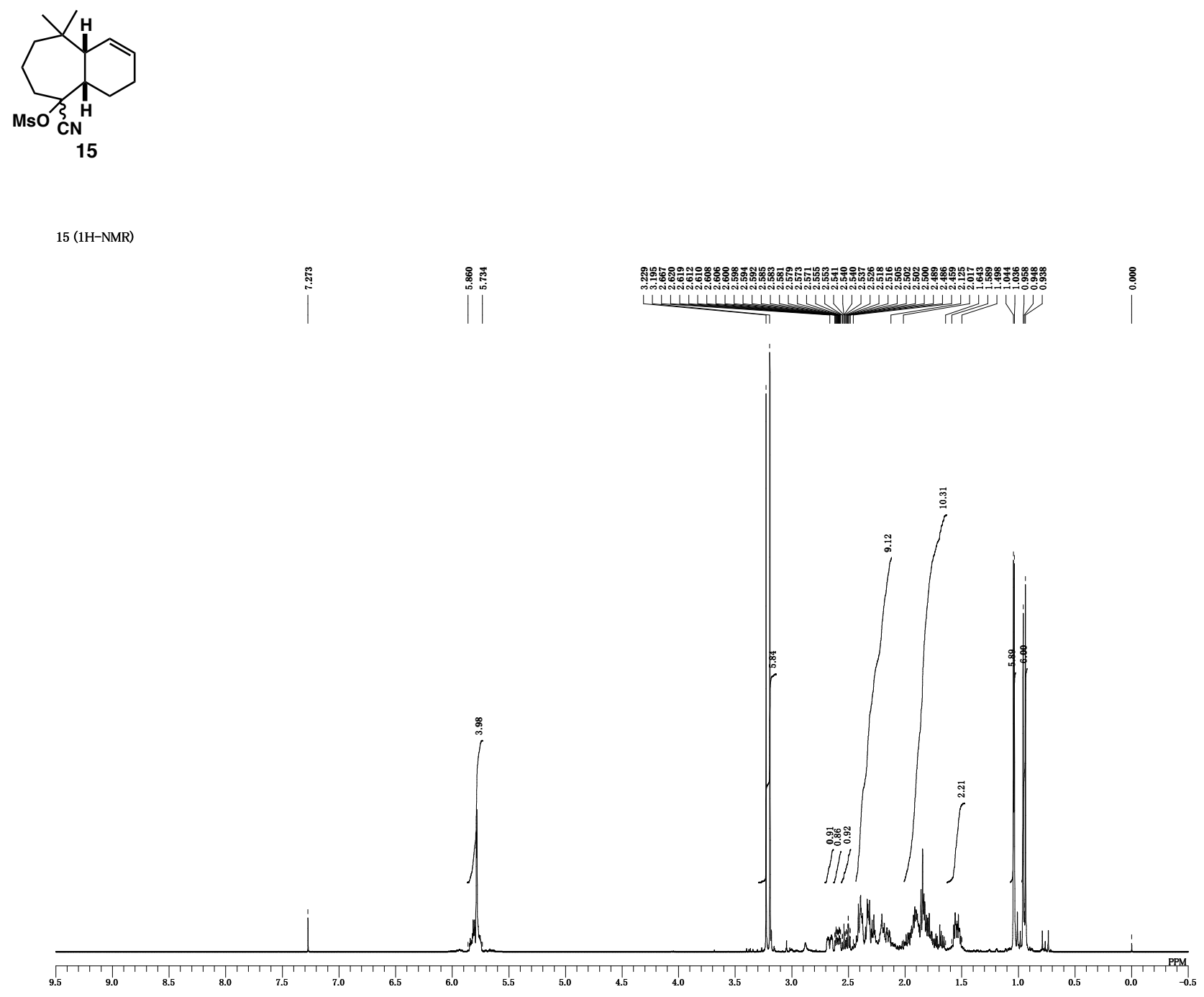

15 (13C-NMR)

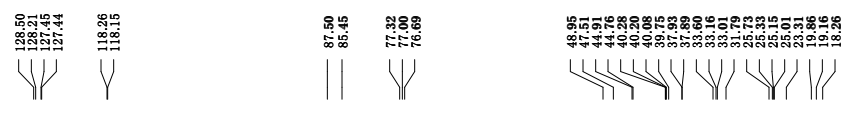

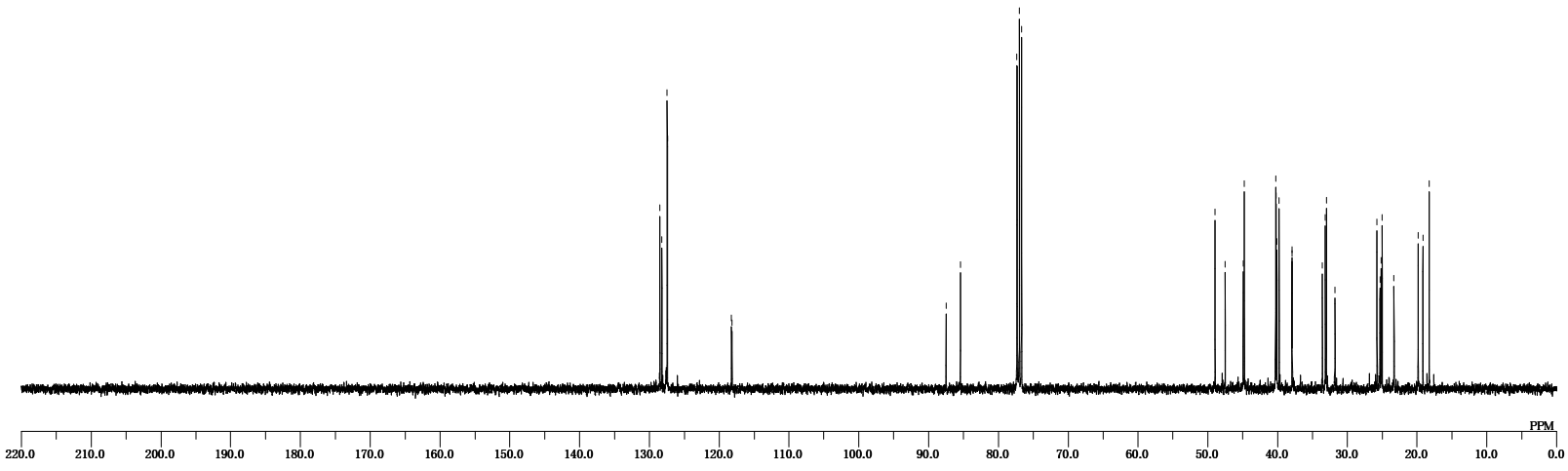


$\underbrace{\mathrm{C}}_{\mathrm{MsO}}$

$7 \beta$ (1H-NMR)

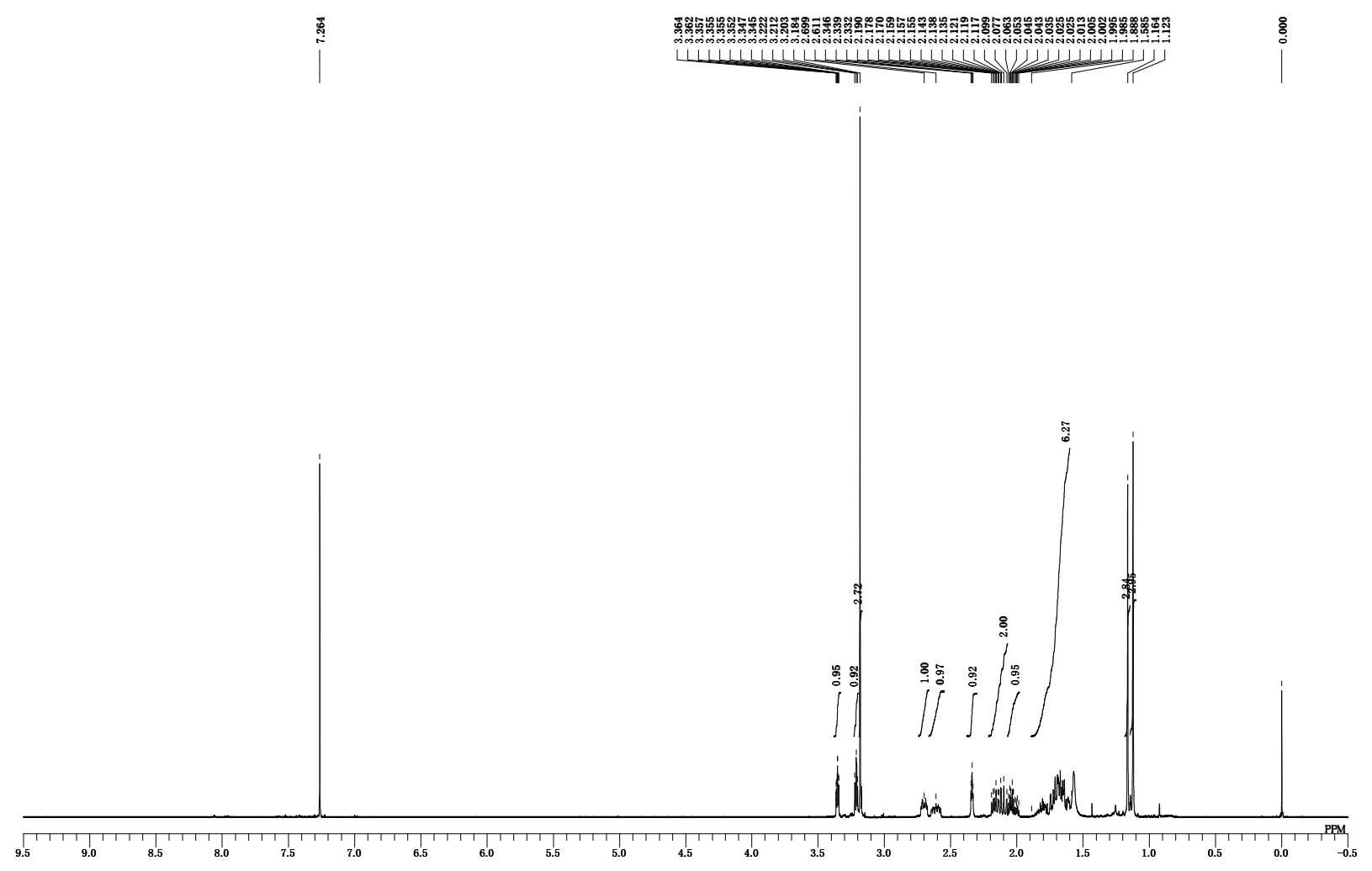

$7 \beta$ (13C-NMR)

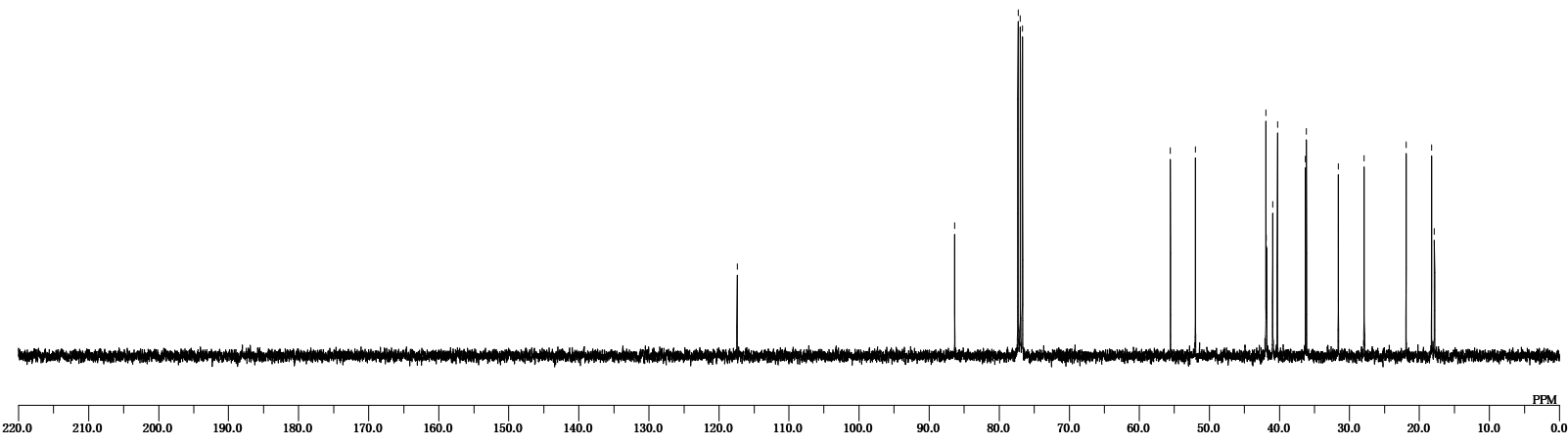




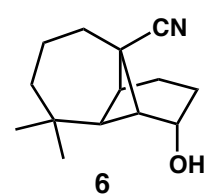

6 (1H-NMR)

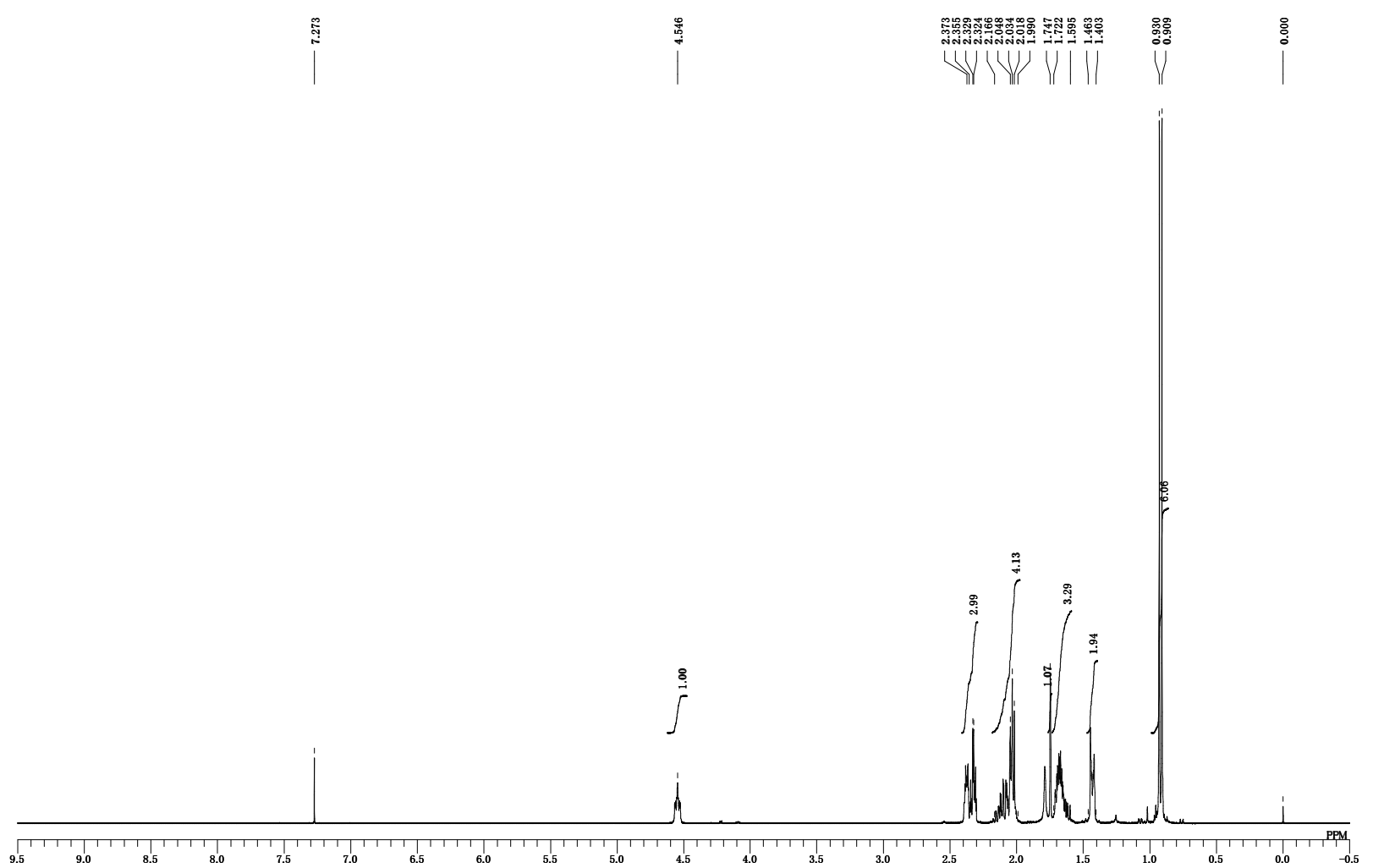

6 (13C-NMR)
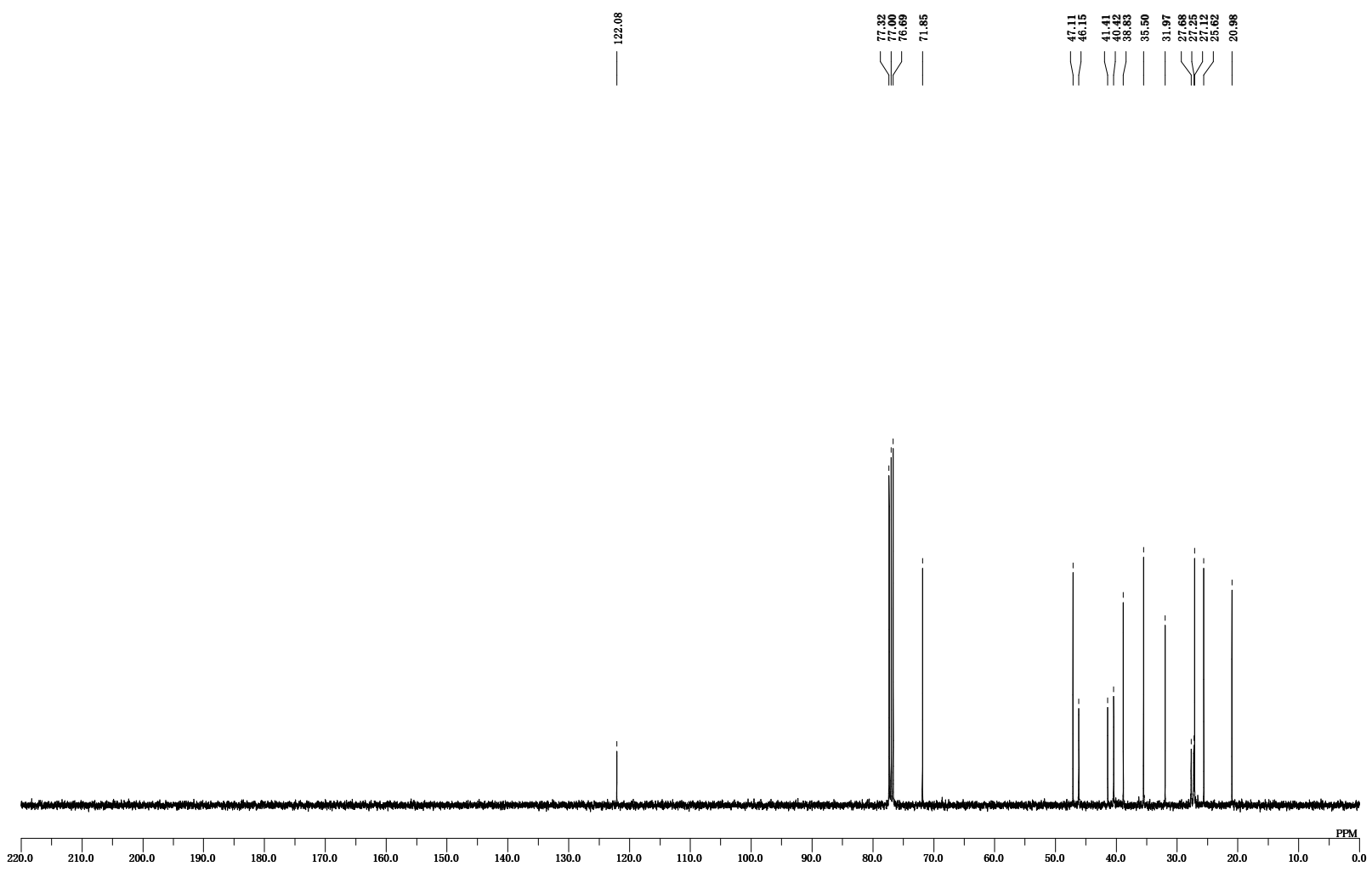


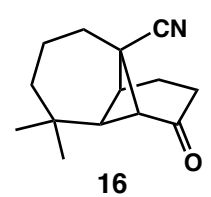

16 (1H-NMR)

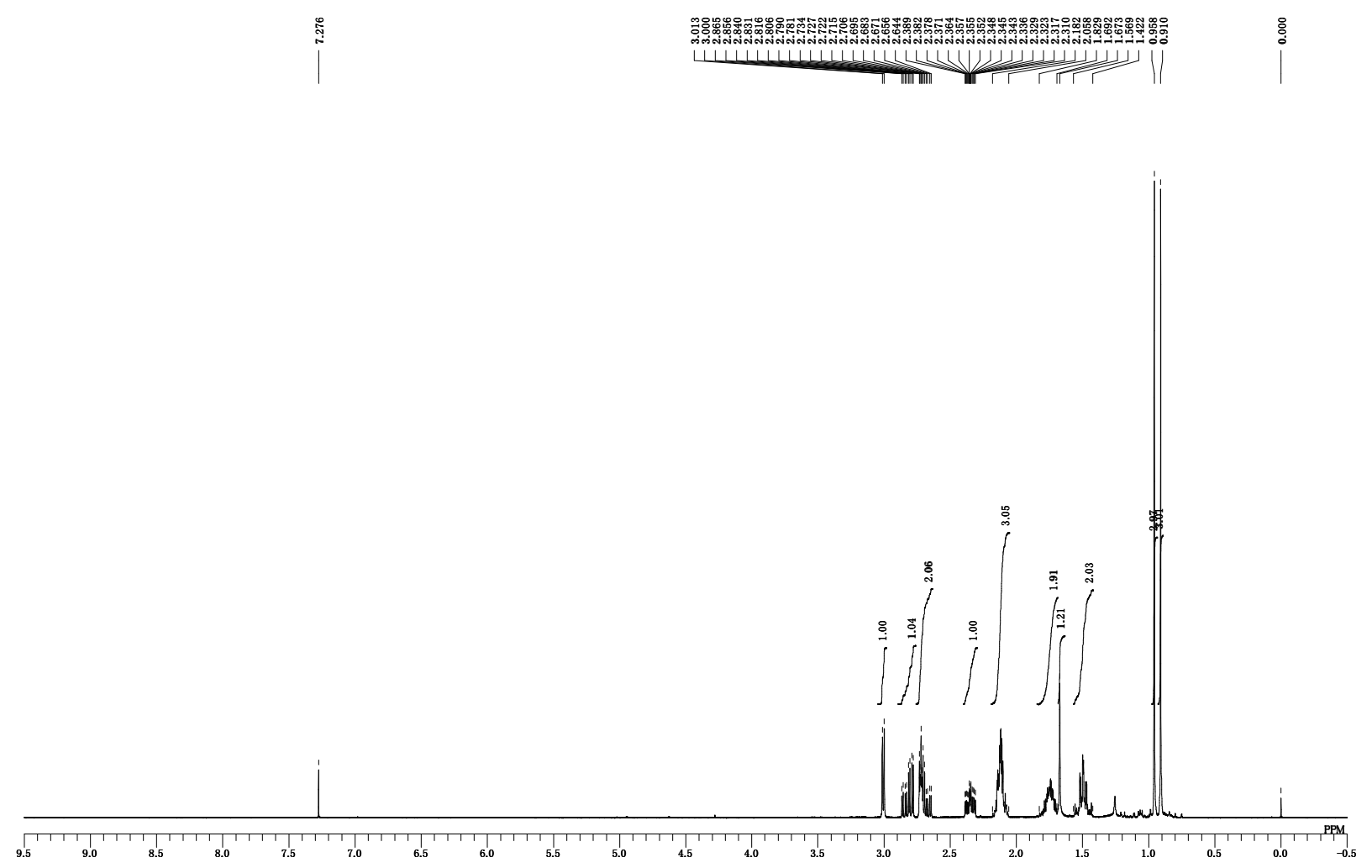

$16(13 \mathrm{C}-\mathrm{NMR})$

|

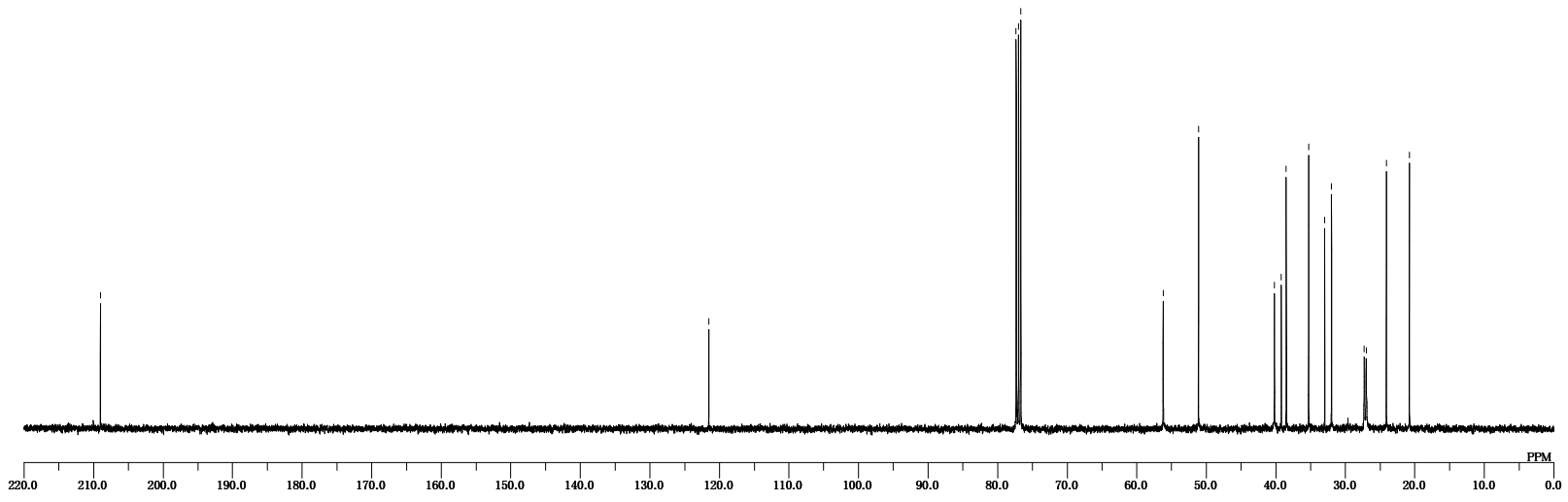




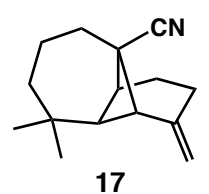

17 (1H-NMR)

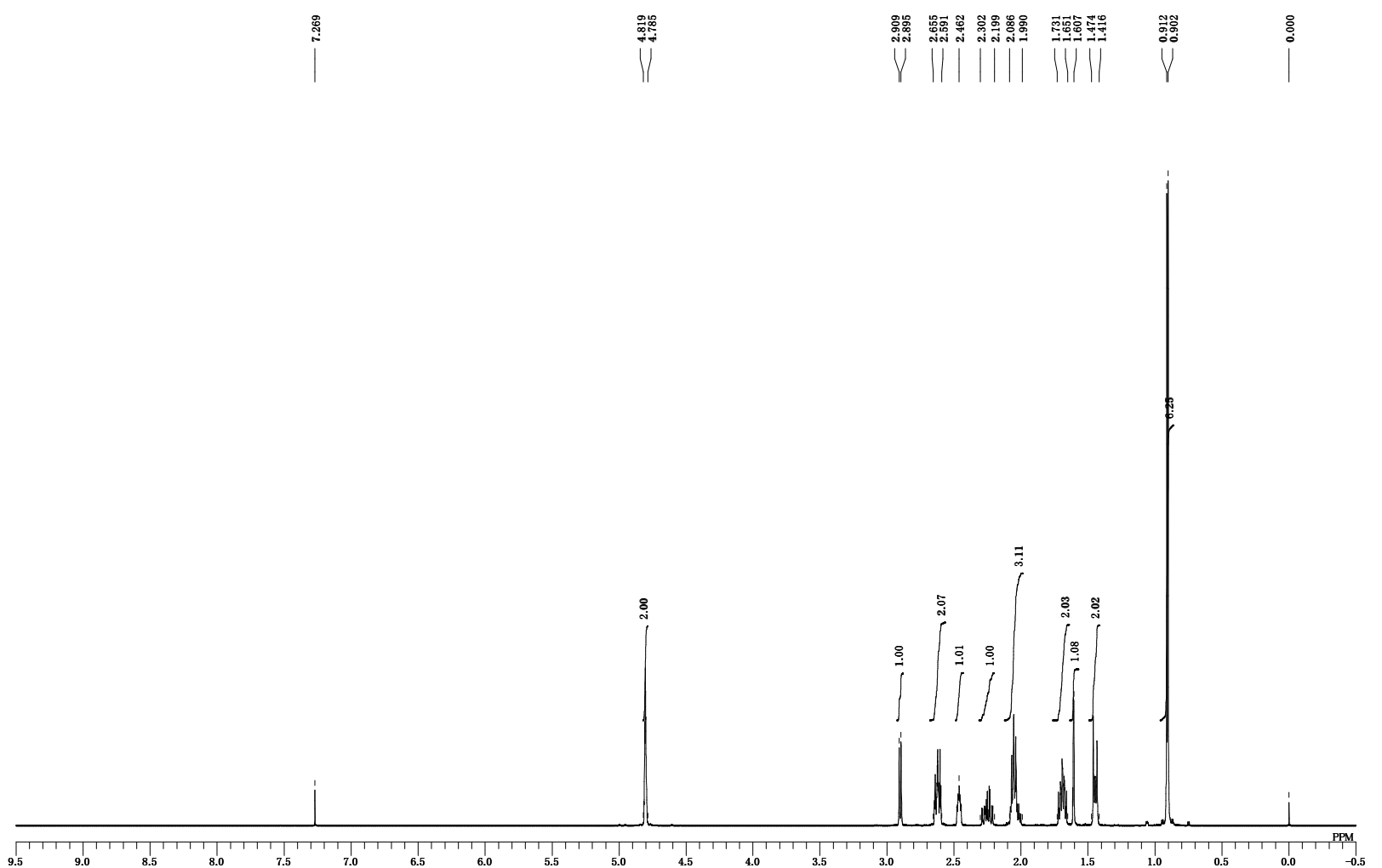

17 (13C-NMR)

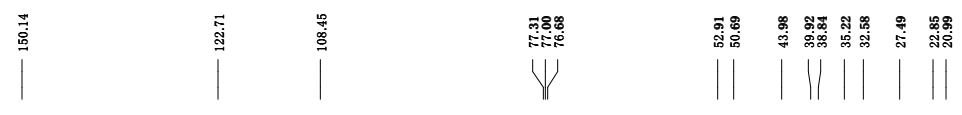

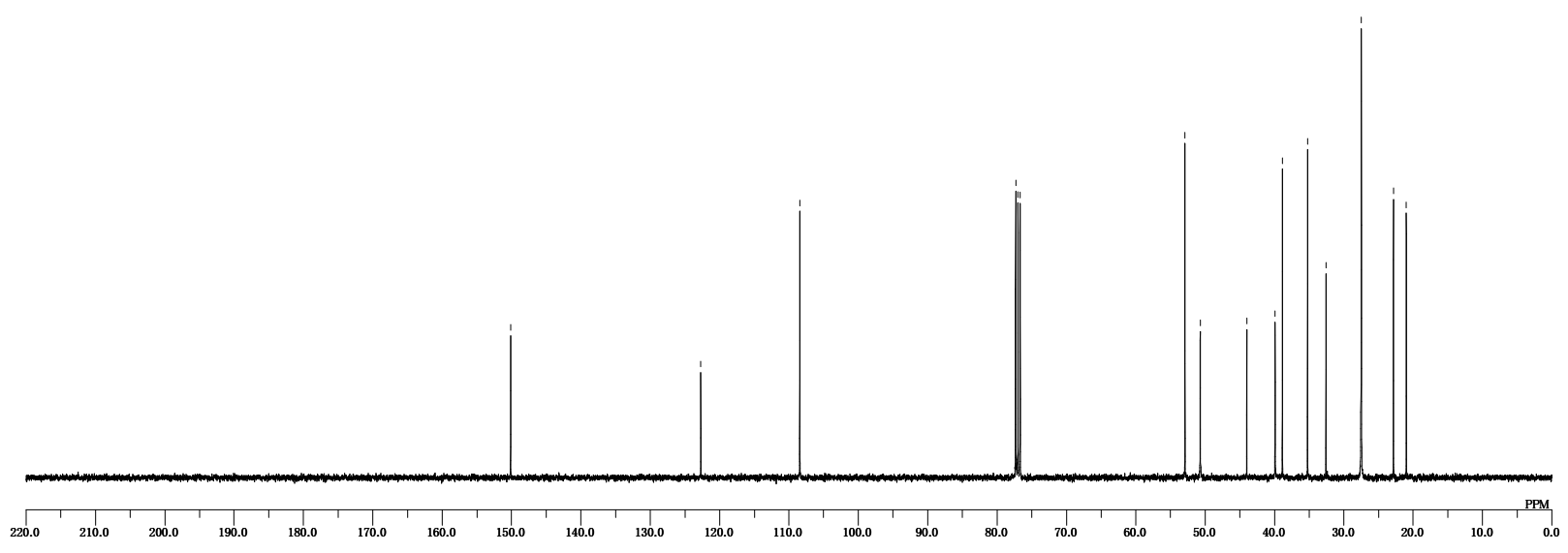




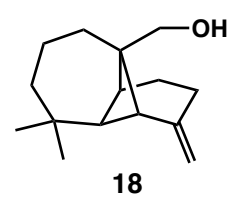

18 (1H-NMR)

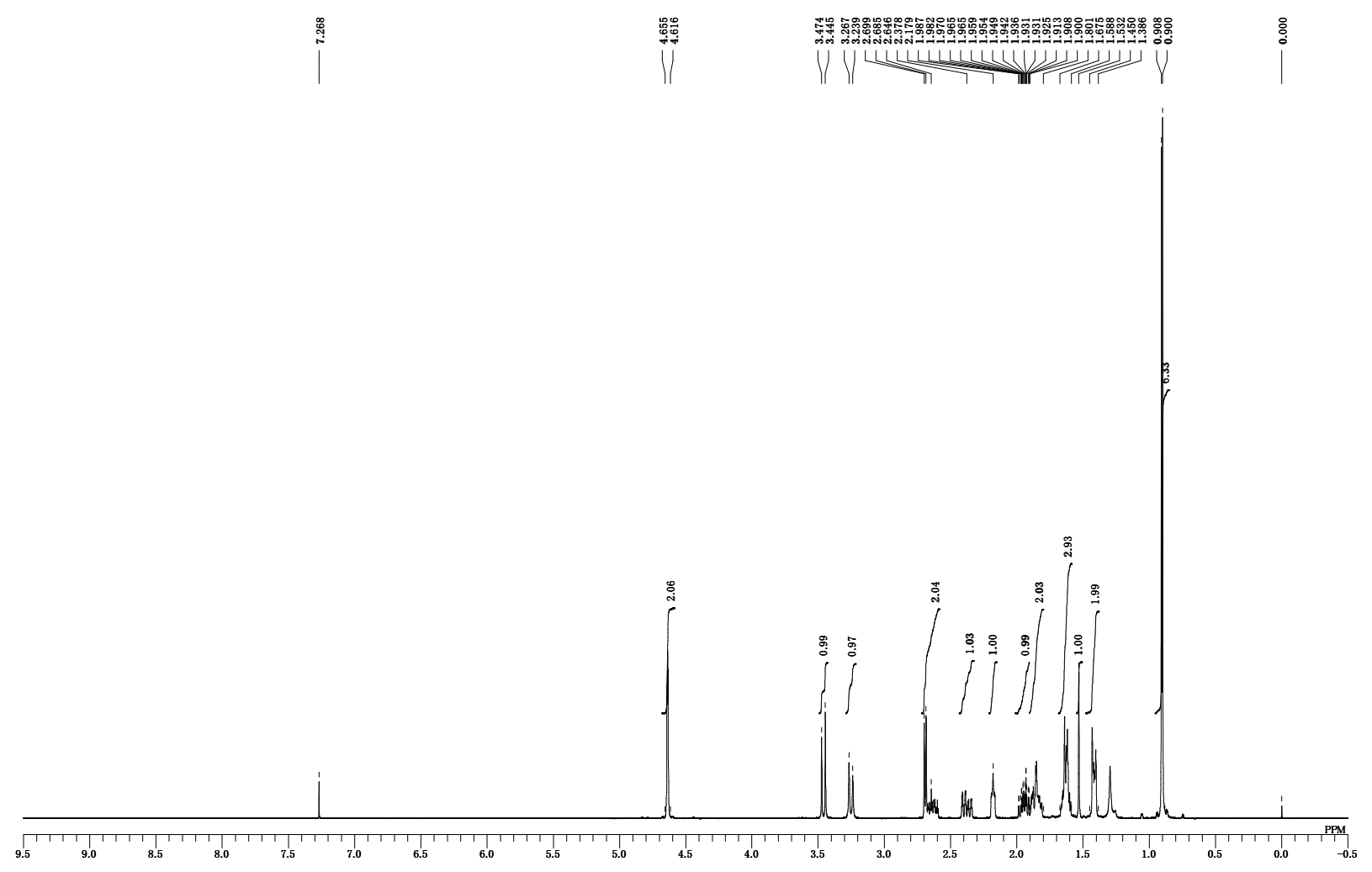

18 (13C-NMR)

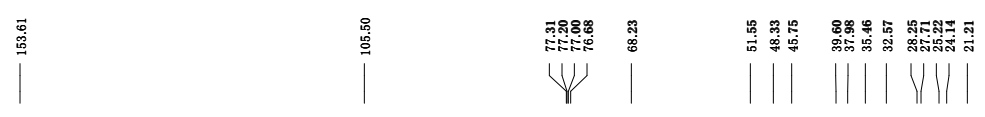

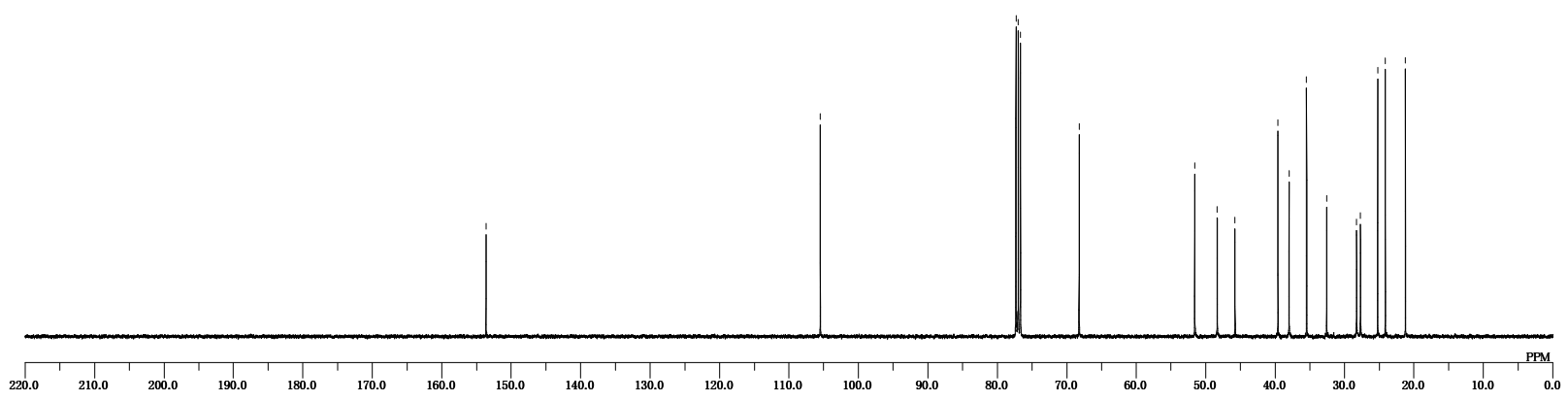




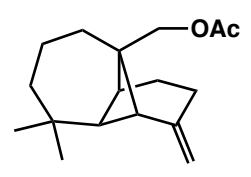

19

19 (1H-NMR)

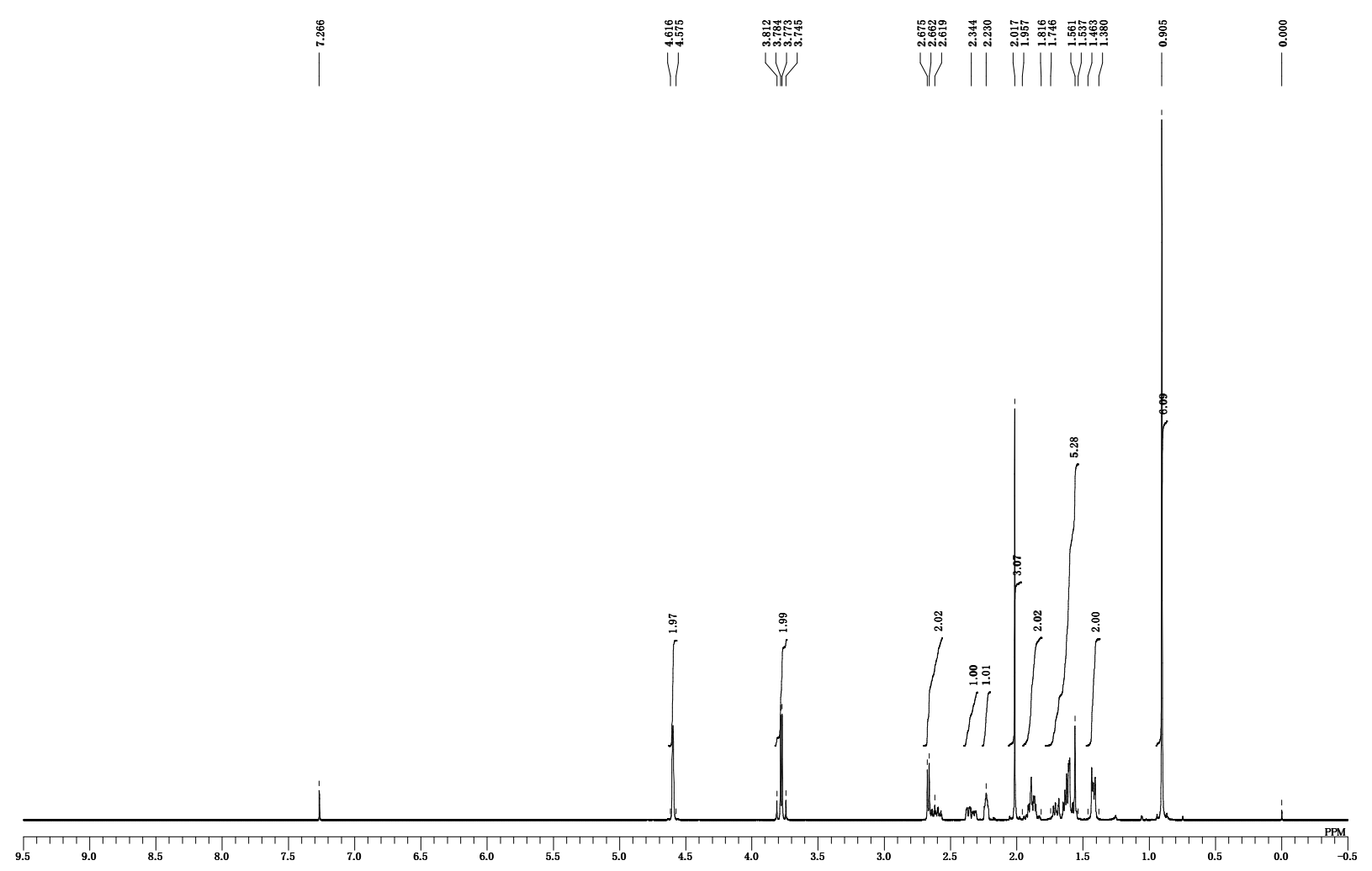

19 (13C-NMR)

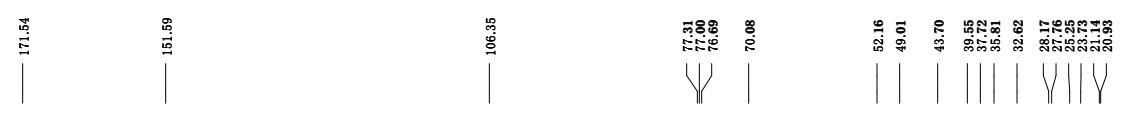




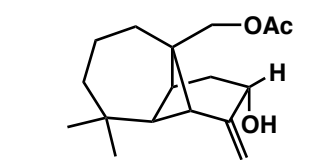

9-acetoxy-3-epi-marsupellol

9-acetoxy-3-epi-marsupellol (1H-NMR)

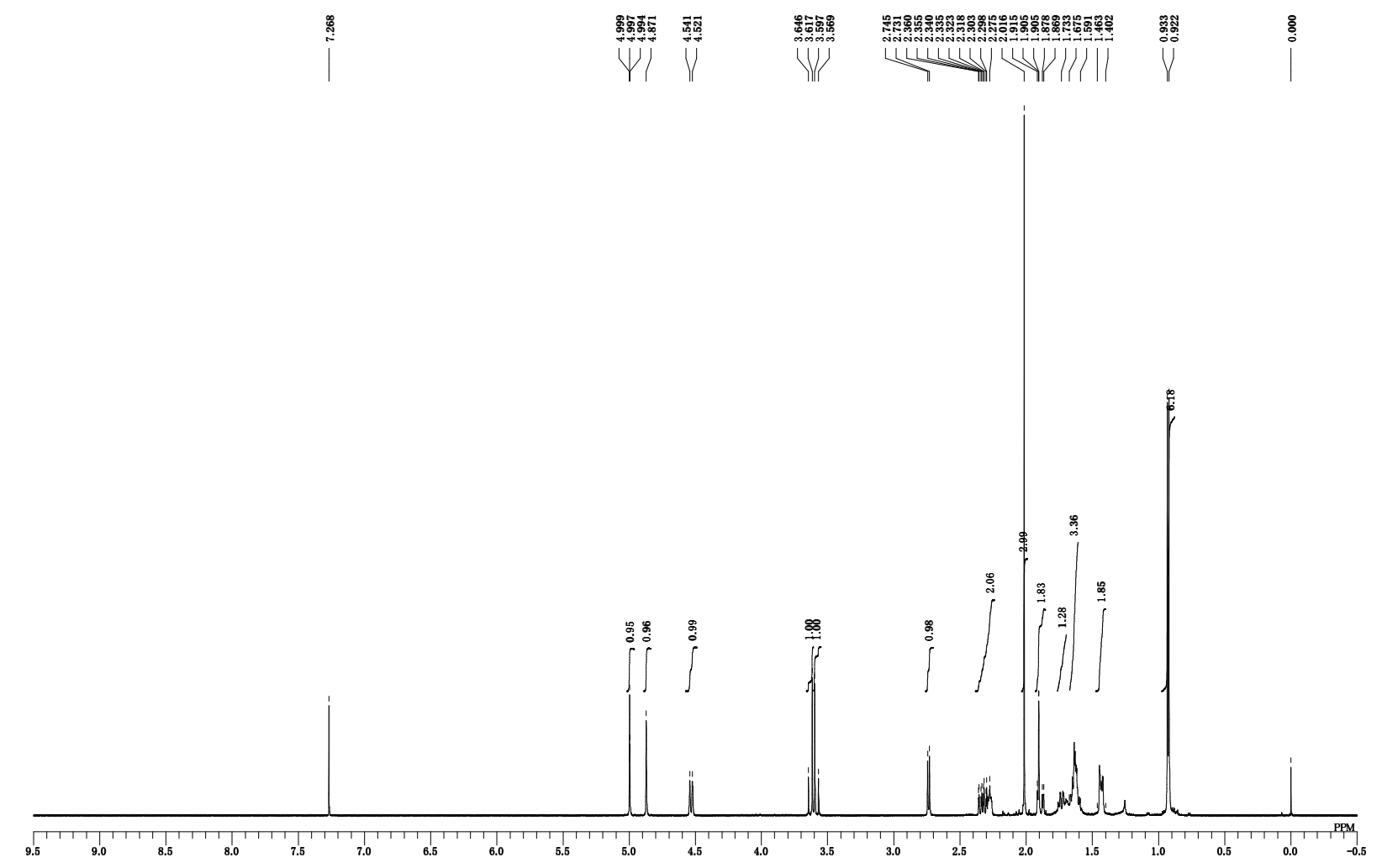

9-acetoxy-3-epi-marsupellol (13C-NMR)
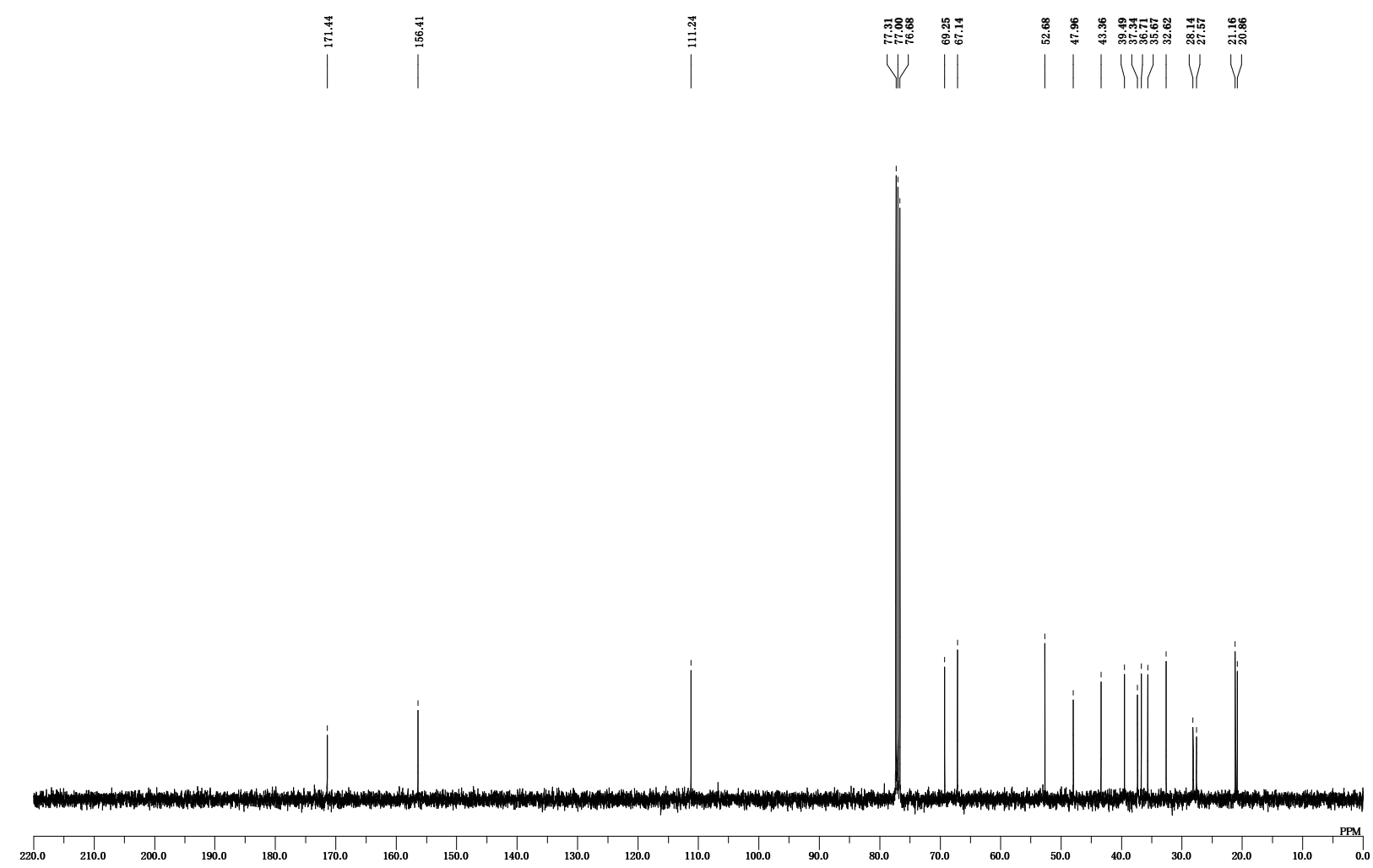


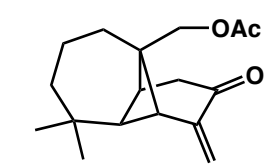

acetoxymarsupellone

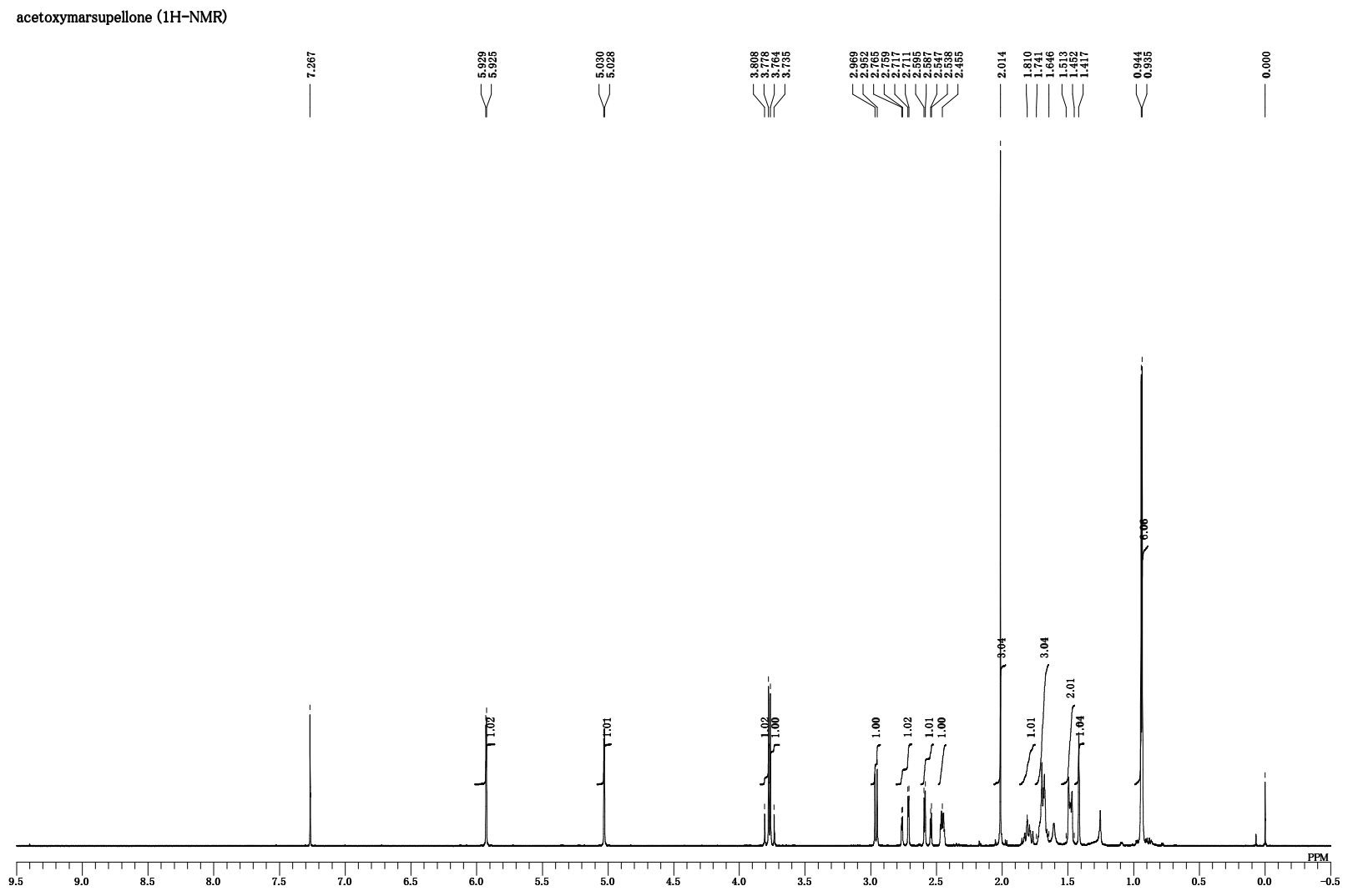

acetoxymarsupellone (13C-NMR)

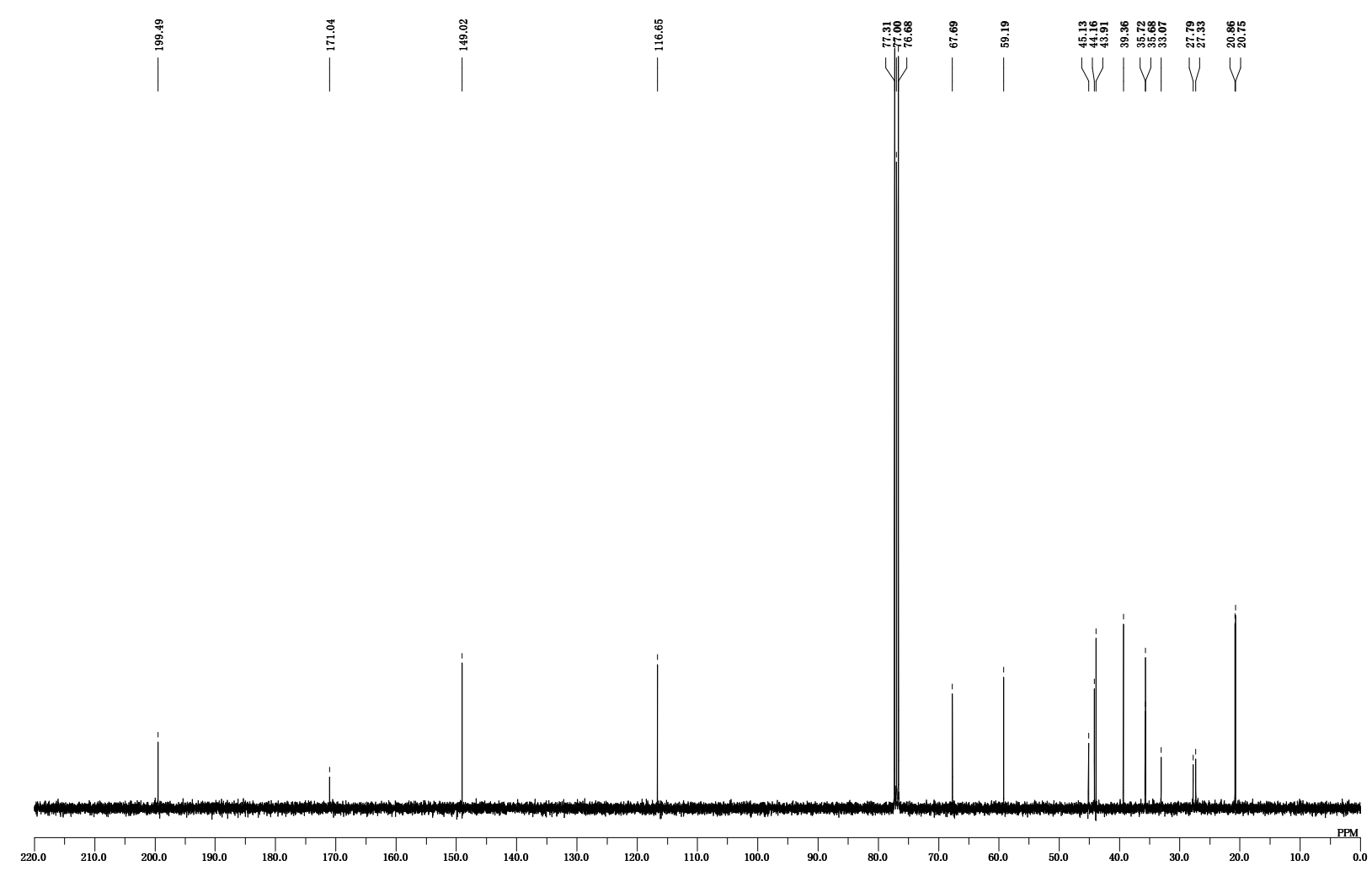




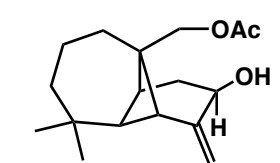

9-acetoxymarsupellol

9-acetoxymarsupellol (1H-NMR)

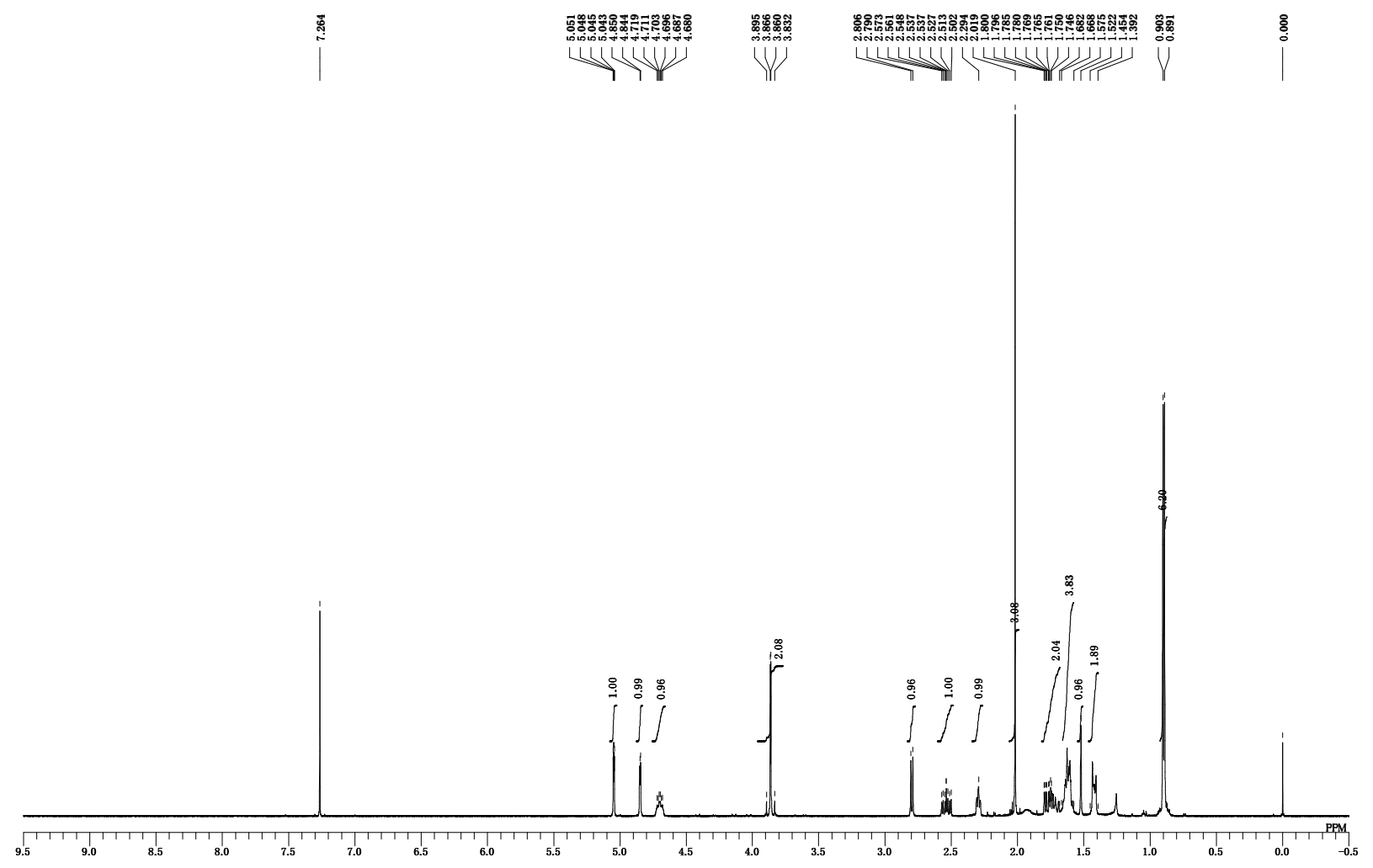

9-acetoxymarsupellol (13C-NMR)

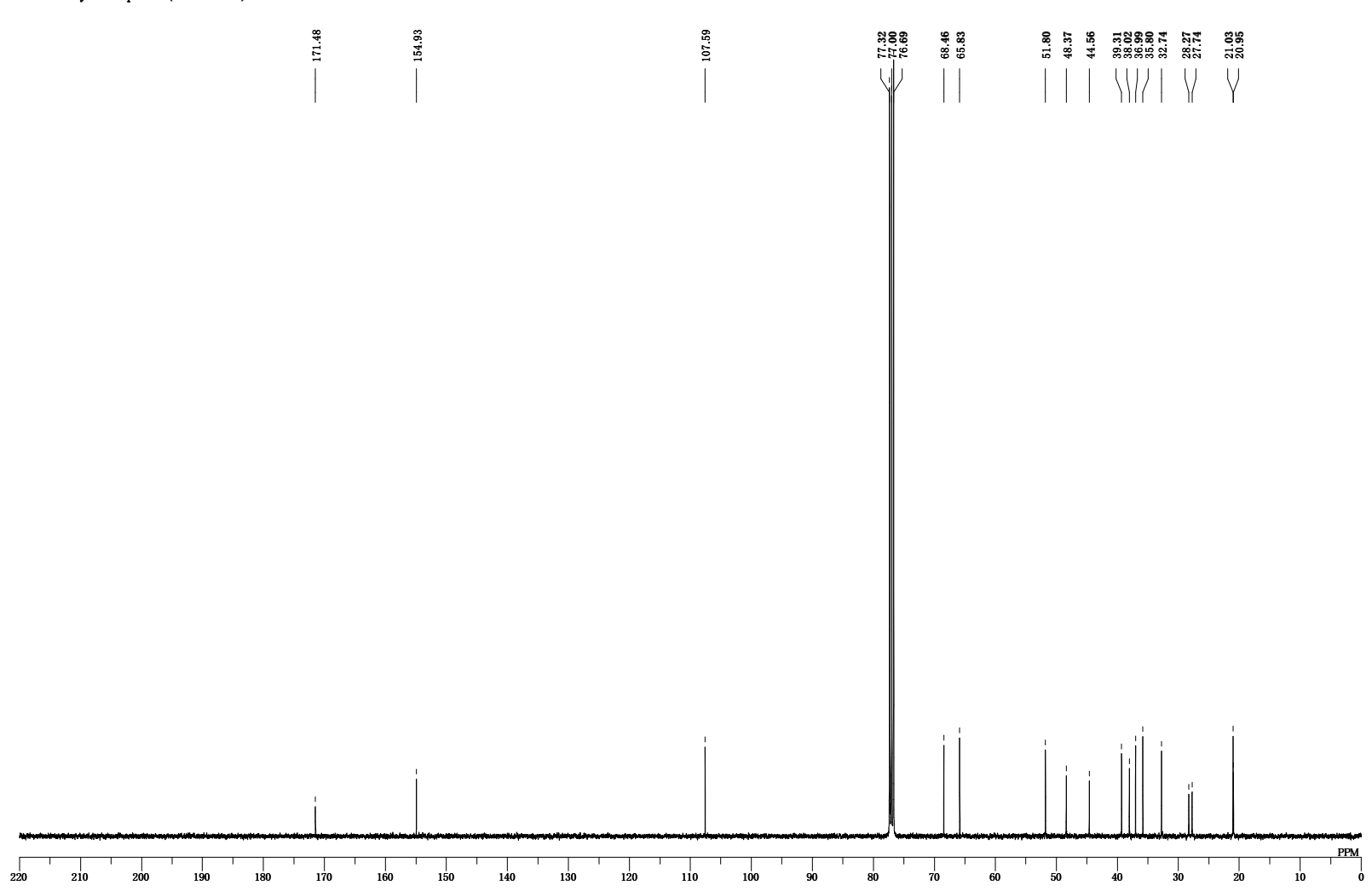




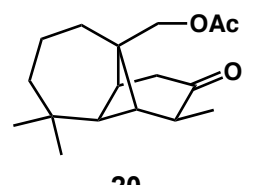

20

20 (1H-NMR)

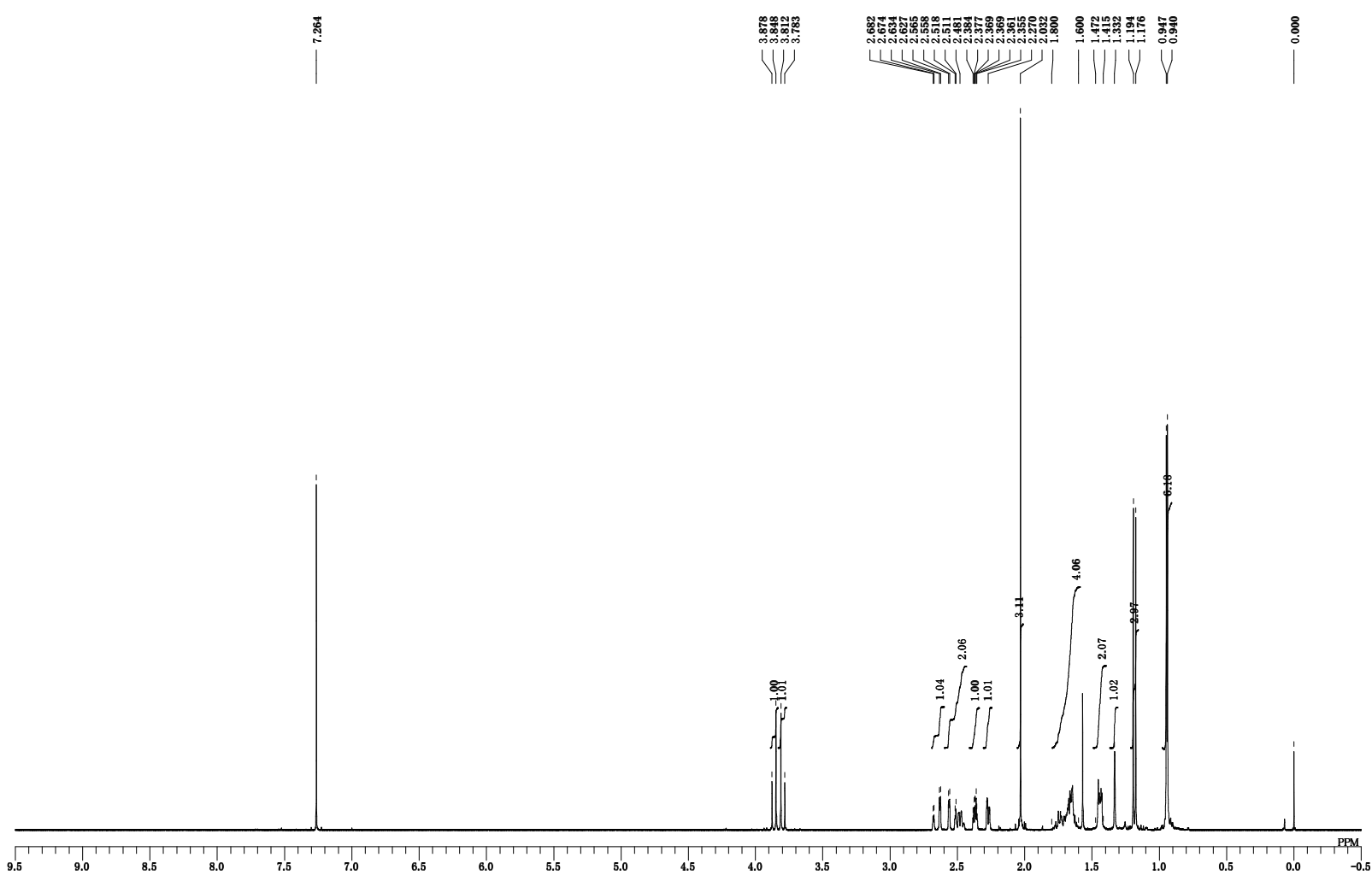




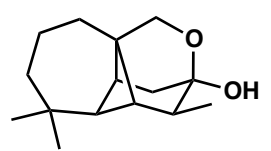

marsupellin B

marsupellin B (1H-NMR)

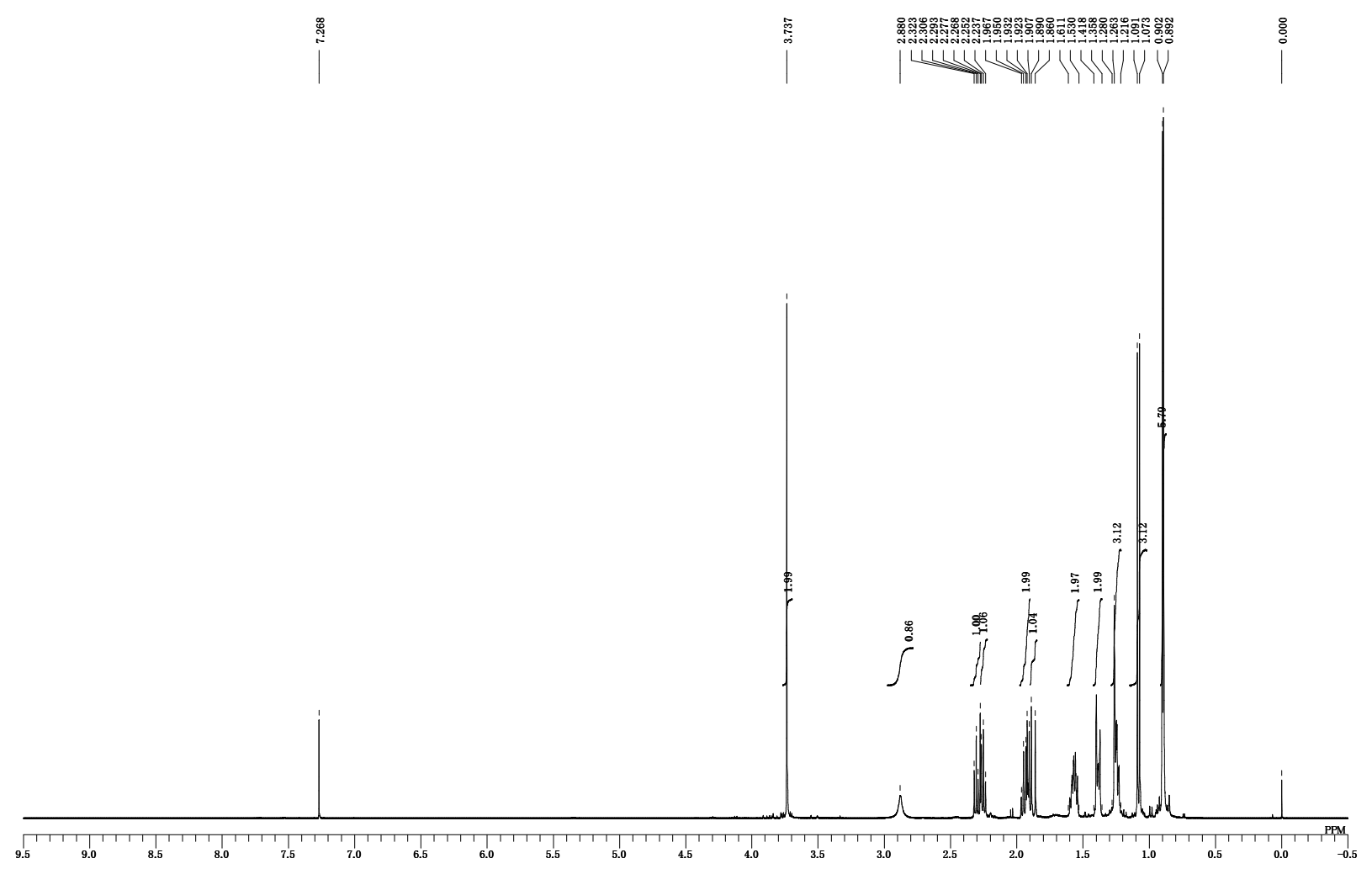

marsupellin B (13C-NMR)

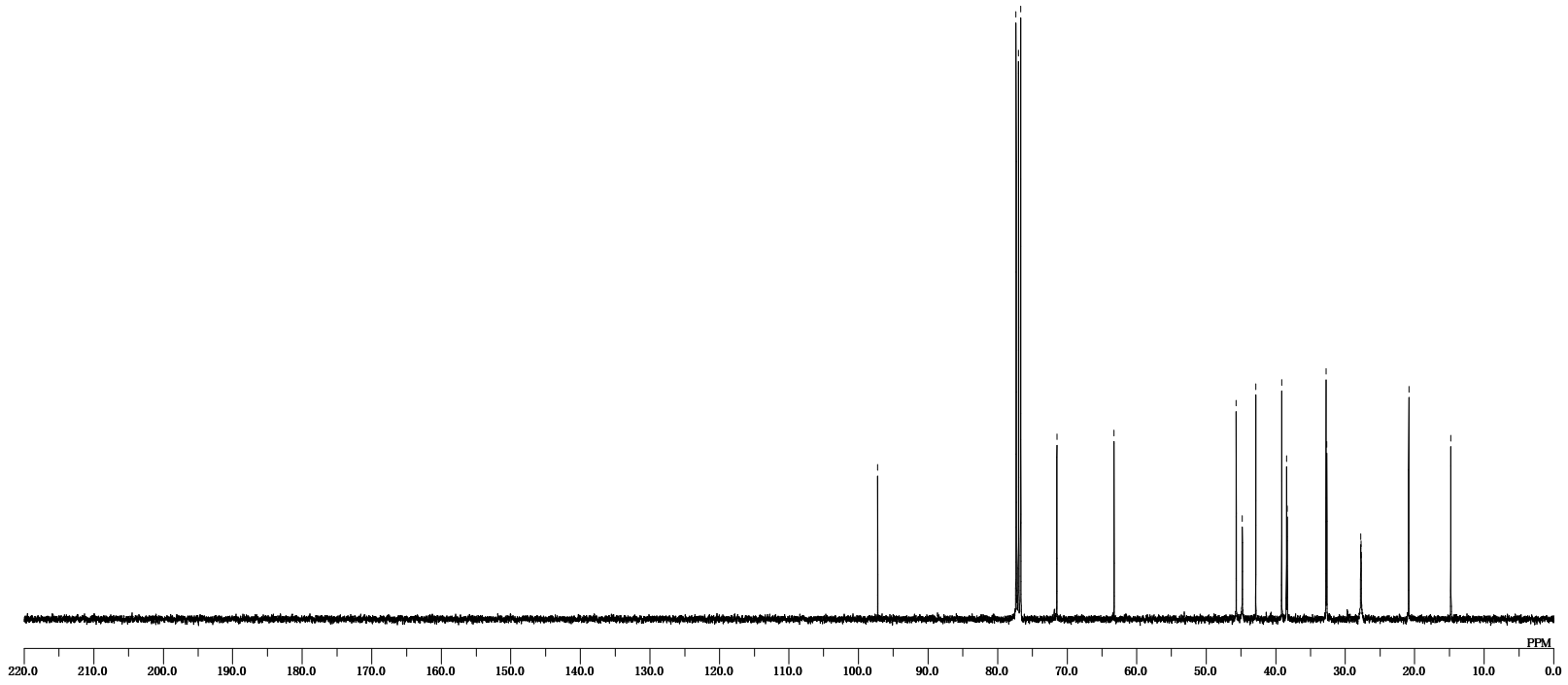




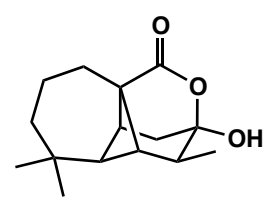

marsupellin A

marsupellin A (1H-NMR)

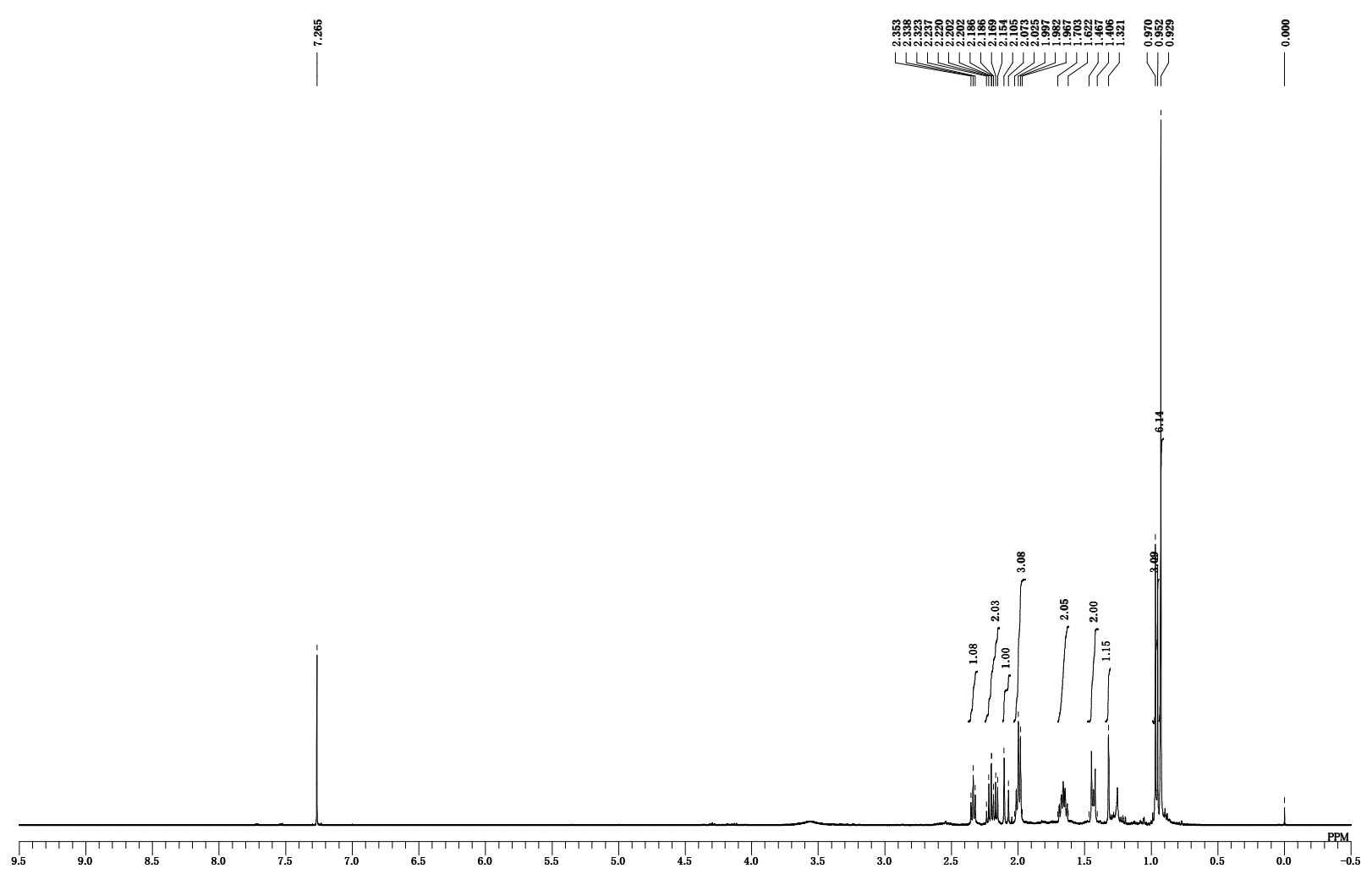

marsupellin A (13C-NMR)

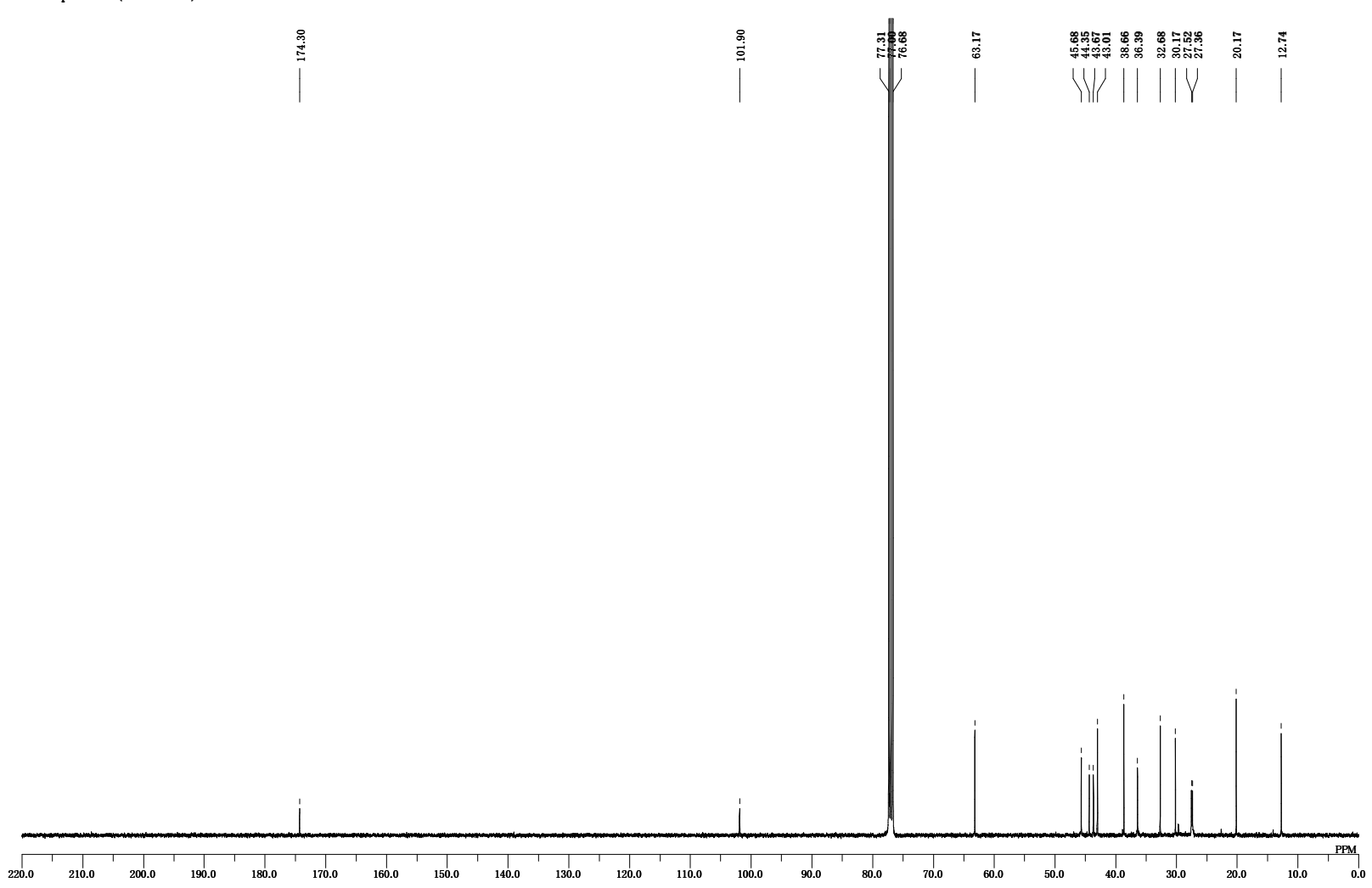

